\title{
Smooth Decompositions of Triebel-Lizorkin and Besov Spaces on Product Spaces of Homogeneous Type
}

\author{
Fanghui Liao, Zongguang Liu, and Xiaojin Zhang \\ Department of Mathematics, China University of Mining \& Technology (Beijing), Beijing 100083, China \\ Correspondence should be addressed to Fanghui Liao; liaofanghui1028@163.com
}

Received 1 March 2014; Revised 28 May 2014; Accepted 16 June 2014; Published 22 July 2014

Academic Editor: Josip E. Pečarić

Copyright (c) 2014 Fanghui Liao et al. This is an open access article distributed under the Creative Commons Attribution License, which permits unrestricted use, distribution, and reproduction in any medium, provided the original work is properly cited.

We introduce Triebel-Lizorkin and Besov spaces by Calderón's reproducing formula on product spaces of homogeneous type. We also obtain smooth atomic and molecular decompositions for these spaces.

\section{Introduction and Main Results}

Atomic and molecular decompositions are significant tools in studying function spaces and operators in harmonic analysis. The atomic decomposition for Hardy spaces on $\mathbb{R}$ was first introduced by Coifman in [1] and was extended to $\mathbb{R}^{n}$ by Latter in [2]. Molecules in Hardy spaces were also introduced by Coifman in [3]. Coifman and Weiss in [4] extended molecules to more general setting in Hardy spaces.

The atomic decomposition of product Hardy spaces $H^{p}(\mathbb{R} \times \mathbb{R})$ was established by Chang and Fefferman in $[5,6]$. Recently, general atomic decomposition on product Hardy spaces was constructed by Han et al. in [7] and Han et al. in [8].

The smooth atomic decomposition for the TriebelLizorkin spaces on $\mathbb{R}^{n}$ first is considered by Frazier and Jawerth in [9] and later extended to spaces of homogeneous type by Han and Sawyer in [10]; for similar results, see [11]. Smooth molecules of the Triebel-Lizorkin spaces on bidisc were given by Wang in [12].

In [13], Hardy spaces associated with different homogeneities were constructed. Wu in [14] introduced related Triebel-Lizorkin and Besov spaces similarly. In $[7,15]$, the authors studied the Hardy spaces on product spaces of homogeneous type and showed some properties of these spaces. A natural question arises: whether we introduce the Triebel-Lizorkin and Besov spaces on product spaces of homogeneous type and study some properties of these spaces.
The purpose of this paper is to answer the question. Namely, we introduce the Triebel-Lizorkin and Besov spaces on product spaces of homogeneous type. A theory of atomic and molecular decompositions for these spaces is also presented.

We begin with some necessary definitions and notation on spaces of homogeneous type.

A quasimetric $\rho$ on a set $X$ is a function $\rho: X \times X \rightarrow$ $[0, \infty)$ satisfying that (i) $\rho(x, y)=0$ if and only if $x=y$, (ii) $\rho(x, y)=\rho(y, x)$ for all $x, y \in X$, and (iii) there exists a constant $A \in[1, \infty)$ such that for all $x, y$, and $z \in X$

$$
\rho(x, y) \leq A[\rho(x, z)+\rho(z, y)] .
$$

Any quasimetric defines a topology, for which the balls $B(x, r)=\{y \in X: \rho(y, x)<r\}$ with all $x \in X$ and all $r>0$ form a basis.

We now state definitions of spaces of homogeneous type.

Definition 1. A space of homogeneous type $(X, \rho, \mu)$ is a set $X$ with a quasimetric $\rho$ and a nonnegative Borel regular measure $\mu$ on $X$ such that $0<\mu(B(x, r))<\infty$, and there exists a positive constant $C<\infty$ such that

$$
\mu(B(x, 2 r)) \leq C \mu(B(x, r))
$$

for all $x \in X$ and all $r>0$, where $\mu$ is assumed to be defined on a $\sigma$-algebra which contains all Borel sets and all balls $B(x, r)$. 
We suppose that $\mu(X)=\infty$ and $\mu(\{x\})=0$ for all $x \in X$. Further, suppose there exist constant $C>0$ and regularity exponent $0<\theta<1$ such that, for all $0<r<\infty$ and all $x, x^{\prime}, y \in X, \mu(B(x, r)) \sim r$ and $\left|\rho(x, y)-\rho\left(x^{\prime}, y\right)\right| \leq$ $C \rho\left(x, x^{\prime}\right)^{\theta}\left[\rho(x, y)+\rho\left(x^{\prime}, y\right)\right]^{1-\theta}$.

Let us recall the definition of an approximation to the identity on spaces of homogeneous type.

Definition 2 (see [7]). Let $X$ be a space of homogeneous type as in Definition 1 and constant $A$ satisfying (1). A sequence $\left\{S_{k}\right\}_{k \in \mathbb{Z}}$ of linear operators is said to be an approximation to the identity of order $\epsilon \in(0, \theta]$ if there exists $C>0$ such that, for all $x, x^{\prime}, y$, and $y^{\prime} \in X, S_{k}(x, y)$, the kernel of $S_{k}$, is a function from $X \times X$ into $\mathbb{C}$ satisfying

(i) $\left|S_{k}(x, y)\right| \leq C\left(2^{-k \epsilon} /\left(2^{-k}+\rho(x, y)\right)^{1+\epsilon}\right)$;

(ii) $\left|S_{k}(x, y)-S_{k}\left(x^{\prime}, y\right)\right| \leq C\left(\rho\left(x, x^{\prime}\right) /\left(2^{-k}+\rho(x\right.\right.$, $y)))^{\epsilon}\left(2^{-k \epsilon} /\left(2^{-k}+\rho(x, y)\right)^{1+\epsilon}\right)$

for $\rho\left(x, x^{\prime}\right) \leq(1 / 2 A)\left(2^{-k}+\rho(x, y)\right)$;

(iii) $\left|S_{k}(x, y)-S_{k}\left(x, y^{\prime}\right)\right| \leq C\left(\rho\left(y, y^{\prime}\right) /\left(2^{-k}+\rho(x\right.\right.$, $y)))^{\epsilon}\left(2^{-k \epsilon} /\left(2^{-k}+\rho(x, y)\right)^{1+\epsilon}\right)$

for $\rho\left(y, y^{\prime}\right) \leq(1 / 2 A)\left(2^{-k}+\rho(x, y)\right)$;

(iv) $\left|\left[S_{k}(x, y)-S_{k}\left(x, y^{\prime}\right)\right]-\left[S_{k}\left(x^{\prime}, y\right)-S_{k}\left(x^{\prime}, y^{\prime}\right)\right]\right|$

$\leq C\left(\rho\left(x, x^{\prime}\right) /\left(2^{-k}+\rho(x, y)\right)\right)^{\epsilon}\left(\rho\left(y, y^{\prime}\right) /\left(2^{-k}+\rho(x\right.\right.$, $y)))^{\epsilon}\left(2^{-k \epsilon} /\left(2^{-k}+\rho(x, y)\right)^{1+\epsilon}\right)$

for $\rho\left(x, x^{\prime}\right) \leq(1 / 2 A)\left(2^{-k}+\rho(x, y)\right)$ and $\rho\left(y, y^{\prime}\right) \leq$ $(1 / 2 A)\left(2^{-k}+\rho(x, y)\right)$;

(v) $\int_{X} S_{k}(x, y) d \mu(y)=\int_{X} S_{k}(x, y) d \mu(x)=1$.

Moreover, a sequence $\left\{S_{k}\right\}_{k \in \mathbb{Z}}$ of linear operators is said to be an approximation to the identity of older $\epsilon \in(0, \theta]$ having compact support if there exists a constant $C>0$ such that, for all $k \in \mathbb{Z}$ and all $x, x^{\prime}, y$, and $y^{\prime} \in X, S_{k}(x, y)$, the kernel of $S_{k}$ is a function from $X \times X$ into $\mathbb{C}$ satisfying $(\mathrm{v})$ and

(vi) $S_{k}(x, y)=0$ if $\rho(x, y) \geq C 2^{-k}$ and $\left\|S_{k}\right\|_{L^{\infty}(X \times X)} \leq C 2^{k}$;

(vii) $\left|S_{k}(x, y)-S_{k}\left(x^{\prime}, y\right)\right| \leq C 2^{k(1+\epsilon)} \rho\left(x, x^{\prime}\right)^{\epsilon}$;

(viii) $\left|S_{k}(x, y)-S_{k}\left(x, y^{\prime}\right)\right| \leq C 2^{k(1+\epsilon)} \rho\left(y, y^{\prime}\right)^{\epsilon}$;

(ix) $\left|\left[S_{k}(x, y)-S_{k}\left(x, y^{\prime}\right)\right]-\left[S_{k}\left(x^{\prime}, y\right)-S_{k}\left(x^{\prime}, y^{\prime}\right)\right]\right| \leq$ $C 2^{k(1+2 \epsilon)} \rho\left(x, x^{\prime}\right)^{\epsilon} \rho\left(y, y^{\prime}\right)^{\epsilon}$.

We present test functions on spaces of homogeneous type $X$ before we give test functions on product spaces of homogeneous type $X_{1} \times X_{2}$.

Definition 3 (see [15]). For fixed $x_{0} \in X$, let $0<\beta, \gamma<\theta$, and $r>0$, where $\theta$ is the regularity exponent on $X$. A function $f(x, y)$ defined on $X \times X$ is said to be a test function of type $\left(x_{0}, r, \beta, \gamma\right)$, if, for all $x, y \in X, f$ satisfies the following conditions:

(i) $|f(x)| \leq C\left(r /\left(r+\rho\left(x, x_{0}\right)\right)\right)^{1+\gamma}$;

(ii) $|f(x)-f(y)| \leq C\left(\rho(x, y) /\left(r+\rho\left(x, x_{0}\right)\right)\right)^{\beta}(r /(r+\rho(x$, $\left.\left.\left.x_{0}\right)\right)\right)^{1+\gamma}$ for $\rho(x, y) \leq(1 / 2 A)\left(r+\rho\left(x, x_{0}\right)\right)$.
If $f$ is a test function of type $\left(x_{0}, r, \beta, \gamma\right)$, we write $f \in$ $\mathscr{G}\left(x_{0}, r, \beta, \gamma\right)$ and the norm of $f$ is defined by

$$
\|f\|_{\mathscr{G}\left(x_{0}, r, \beta, \gamma\right)}=\inf \{C: \text { (i)-(ii) hold }\} .
$$

We denote by $\mathscr{G}(\beta, \gamma)$ the class of $\mathscr{G}\left(x_{0}, r, \beta, \gamma\right)$ with $r=1$ for fixed $x_{0} \in X$. Set $f(x) \in \mathscr{G}_{0}(\beta, \gamma)$ if $f(x) \in \mathscr{G}(\beta, \gamma)$ with $\int_{X} f(x) d \mu(x)=0$. It is easy to see that $\mathscr{G}\left(x_{1}, r, \beta, \gamma\right)=$ $\mathscr{G}(\beta, \gamma)$ with an equivalent norm for all $x_{1} \in X$ and $r>0$. Furthermore, we can check that $\mathscr{G}(\beta, \gamma)$ is a Banach space with respect to the norm in $\mathscr{G}(\beta, \gamma)$. Let $\mathscr{G}^{\circ}(\beta, \gamma)$ be the completion of the space $\mathscr{G}_{0}(\theta, \theta)$ in $\mathscr{G}(\beta, \gamma)$ with $0<\beta, \gamma<\theta$. If $f \in \mathscr{G}(\beta, \gamma)$, we then define $\|f\|_{\mathscr{G}^{\circ}(\beta, \gamma)}=\|f\|_{\mathscr{G}(\beta, \gamma)}$.

We define the distribution space $\left(\mathscr{G}^{\circ}(\beta, \gamma)\right)^{\prime}$ by all linear functionals $\mathscr{L}$ from $\mathscr{G}^{\circ}(\beta, \gamma)$ to $\mathbb{C}$ with the property that there exists $C \geq 0$ such that $|\mathscr{L}(f)| \leq C\|f\|_{\mathscr{G}^{\circ}(\beta, \gamma)}$ for all $f \in$ $\mathscr{G}^{\circ}(\beta, \gamma)$.

Let $\left(X_{i}, \rho_{i}, \mu_{i}\right)$ for $i=1,2$ be two spaces of homogeneous type as in Definition 1 and $\rho_{i}$ satisfies (1) with $A$ replaced by $A_{i}$ for $i=1,2$. We define spaces of test functions on product space $X_{1} \times X_{2}$ of spaces of homogeneous type.

Definition 4 (see [15]). Let $\left(x_{0}, y_{0}\right) \in X_{1} \times X_{2}, 0<\beta_{i}, \gamma_{i}<\theta_{i}$, and $r_{i}>0$, where $\theta_{i}$ is the regularity exponent on $X_{i}$ for $i=1,2$. A function $f(x, y)$ defined on $X_{1} \times X_{2}$ is said to be a test function of type $\left(x_{0}, y_{0} ; r_{1}, r_{2} ; \beta_{1}, \beta_{2} ; \gamma_{1}, \gamma_{2}\right)$ if, for any fixed $y, y^{\prime} \in X_{2}, f(x, y)$, as a function of variable of $x$, is a test function of $\mathscr{C}\left(x_{0}, r_{1}, \beta_{1}, \gamma_{1}\right)$ on $X_{1}$. Similarly, for any fixed $x, x^{\prime} \in X_{1}, f(x, y)$, as a function of variable of $y$, is a test function of $\mathscr{G}\left(y_{0}, r_{2}, \beta_{2}, \gamma_{2}\right)$ on $X_{2}$. Moreover the following conditions are satisfied:

(i) $\|f(\cdot, y)\|_{\mathscr{G}\left(x_{0}, r_{1}, \beta_{1}, \gamma_{1}\right)} \leq C\left(r_{2} /\left(r_{2}+\rho_{2}\left(y, y_{0}\right)\right)\right)^{1+\gamma_{2}}$;

(ii) $\left\|f(\cdot, y)-f\left(\cdot, y^{\prime}\right)\right\|_{\mathscr{G}\left(x_{0}, r_{1}, \beta_{1}, \gamma_{1}\right)} \leq C\left(\rho_{2}\left(y^{\prime}, y\right) /\left(r_{2}+\right.\right.$ $\left.\left.\rho_{2}\left(y, y_{0}\right)\right)\right)^{\beta_{2}}\left(r_{2} /\left(r_{2}+\rho_{2}\left(y, y_{0}\right)\right)\right)^{1+\gamma_{2}}$ for all $y, y^{\prime} \in X_{2}$ with $\rho_{2}\left(y, y^{\prime}\right) \leq\left(1 / 2 A_{2}\right)\left(r_{2}+\rho_{2}\left(y, y_{0}\right)\right)$;

(iii) properties (i)-(ii) also hold with $x$ and $y$ interchanged.

If $f$ is a test function of type $\left(x_{0}, y_{0} ; r_{1}, r_{2} ; \beta_{1}, \beta_{2} ; \gamma_{1}, \gamma_{2}\right)$, we write

$$
f \in \mathscr{G}\left(x_{0}, y_{0} ; r_{1}, r_{2} ; \beta_{1}, \beta_{2} ; \gamma_{1}, \gamma_{2}\right) \text {, }
$$

and the norm of $f$ is defined by

$$
\|f\|_{\mathscr{G}\left(x_{0}, y_{0} ; r_{1}, r_{2} ; \beta_{1}, \beta_{2} ; \gamma_{1}, \gamma_{2}\right)}=\inf \{C: \text { (i) - (iii) hold }\} \text {. }
$$

Similarly, we denote by $\mathscr{E}\left(\beta_{1}, \beta_{2} ; \gamma_{1}, \gamma_{2}\right)$ the class of $\mathscr{G}\left(x_{0}, y_{0} ; r_{1}, r_{2} ; \beta_{1}, \beta_{2} ; \gamma_{1}, \gamma_{2}\right)$ with $r_{1}=r_{2}=1$ for fixed $\left(x_{0}, y_{0}\right) \in X_{1} \times X_{2}$. Set $f(x, y) \in \mathscr{G}_{0}\left(\beta_{1}, \beta_{2} ; \gamma_{1}, \gamma_{2}\right)$ if $f(x, y) \in \mathscr{G}\left(\beta_{1}, \beta_{2} ; \gamma_{1}, \gamma_{2}\right)$ with $\int_{X_{1}} f(x, y) d \mu_{1}(x)=$ $\int_{X_{2}} f(x, y) d \mu_{2}(y)=0$. It is easy to see that

$$
\mathscr{G}\left(x_{0}, y_{0} ; r_{1}, r_{2} ; \beta_{1}, \beta_{2} ; \gamma_{1}, \gamma_{2}\right)=\mathscr{G}\left(\beta_{1}, \beta_{2} ; \gamma_{1}, \gamma_{2}\right)
$$

with an equivalent norm for all $\left(x_{0}, y_{0}\right) \in X_{1} \times X_{2}$ and $r_{1}, r_{2}>$ 0 . Furthermore, we can check that $\mathscr{G}\left(\beta_{1}, \beta_{2} ; \gamma_{1}, \gamma_{2}\right)$ is a Banach space with respect to the norm in $\mathscr{C}\left(\beta_{1}, \beta_{2} ; \gamma_{1}, \gamma_{2}\right)$. 
Let $\mathscr{G}^{\circ}\left(\beta_{1}, \beta_{2}, \gamma_{1}, \gamma_{2}\right)$ be the completion of the space $\mathscr{G}_{0}\left(\theta_{1}, \theta_{2} ; \theta_{1}, \theta_{2}\right)$ in $\mathscr{G}\left(\beta_{1}, \beta_{2}, \gamma_{1}, \gamma_{2}\right)$ with $0<\beta_{i}, \gamma_{i}<\theta_{i}$ for $i=$ 1,2 . If $f \in \mathscr{G}\left(\beta_{1}, \beta_{2} ; \gamma_{1}, \gamma_{2}\right)$, we then define $\|f\|_{\mathscr{C}^{\circ}\left(\beta_{1}, \beta_{2}, \gamma_{1}, \gamma_{2}\right)}=$ $\|f\|_{\mathscr{G}\left(\beta_{1}, \beta_{2}, \gamma_{1}, \gamma_{2}\right)}$.

Also, we define the distribution space $\left(\mathscr{G}^{\circ}\left(\beta_{1}, \beta_{2} ; \gamma_{1}, \gamma_{2}\right)\right)^{\prime}$ by all linear functionals $\mathscr{L}$ from $\mathscr{G}^{\circ}\left(\beta_{1}, \beta_{2} ; \gamma_{1}, \gamma_{2}\right)$ to $\mathbb{C}$ with the property that there exists a constant $C>0$ such that $|\mathscr{L}(f)| \leq C\|f\|_{\mathscr{G}^{\circ}\left(\beta_{1}, \beta_{2} ; \gamma_{1}, \gamma_{2}\right)}$ for all $f \in \mathscr{G}^{\circ}\left(\beta_{1}, \beta_{2} ; \gamma_{1}, \gamma_{2}\right)$.

We give Calderón's reproducing formulas on product spaces of homogeneous type.

Lemma 5 (see $[7,15])$. Suppose that $i=1,2$. Let $\epsilon_{i} \in\left(0, \theta_{i}\right]$, let $\left\{S_{k_{i}}\right\}_{k_{i} \in \mathbb{Z}}$ be an approximation to the identity of order $\epsilon_{i}$ on spaces of homogeneous type $X_{i}$, and let $D_{k_{i}}=S_{k_{i}}-S_{k_{i}-1}$ for $k_{i} \in \mathbb{Z}$. Then there are families of linear operators $\left\{\widetilde{D}_{k_{i}}\right\}_{k_{i} \in \mathbb{Z}}$ and $\left\{\bar{D}_{k_{i}}\right\}_{k_{i} \in \mathbb{Z}}$ on $X_{i}$ such that, for all $f \in\left(\mathscr{G}^{\circ}\left(\beta_{1}, \beta_{2} ; \gamma_{1}, \gamma_{2}\right)\right)^{\prime}$ with $\beta_{i}, \gamma_{i} \in\left(0, \epsilon_{i}\right)$,

$$
f=\sum_{k_{1}, k_{2} \in \mathbb{Z}} \widetilde{D}_{k_{1}} \widetilde{D}_{k_{2}} D_{k_{1}} D_{k_{2}}(f)=\sum_{k_{1}, k_{2} \in \mathbb{Z}} D_{k_{1}} D_{k_{2}} \bar{D}_{k_{1}} \bar{D}_{k_{2}}(f),
$$

where the series converges in the norm of both the spaces $\left(\mathscr{G}^{\circ}\left(\beta_{1}^{\prime}, \beta_{2}^{\prime} ; \gamma_{1}^{\prime}, \gamma_{2}^{\prime}\right)\right)^{\prime}$ with $\beta_{i}^{\prime} \in\left(0, \beta_{i}\right), \gamma_{i}^{\prime} \in\left(0, \gamma_{i}\right)$. Moreover, for $x_{i}, y_{i} \in X_{i}$ and all $k_{i} \in \mathbb{Z}, \widetilde{D}_{k_{i}}\left(x_{i}, y_{i}\right)$, the kernel of $\widetilde{D}_{k_{i}}$, satisfies conditions (i) and (ii) in Definition 2 with $\epsilon_{i}$ replaced by any $\epsilon_{i}^{\prime} \in\left(0, \epsilon_{i}\right)$ and

$$
\int_{X_{i}} \widetilde{D}_{k_{i}}\left(x_{i}, y_{i}\right) d \mu_{i}\left(y_{i}\right)=\int_{X_{i}} \widetilde{D}_{k_{i}}\left(x_{i}, y_{i}\right) d \mu_{i}\left(x_{i}\right)=0 .
$$

$\bar{D}_{k}(x, y)$, the kernel of $\bar{D}_{k}$, satisfies conditions (i) and (iii) in Definition 2 with $\epsilon$ replaced by $\epsilon^{\prime}$ for $0<\epsilon^{\prime}<\epsilon$ and (8).

We also need the following result, which gives an analogue of the grid Euclidean dyadic cubes on a space of homogeneous type.

Lemma 6 (see [15]). Let $X$ be a space of homogeneous type as in Definition 1. Then there exists a collection $\left\{Q_{\alpha}^{k} \subset X: k \in\right.$ $\left.\mathbb{Z}, \alpha \in I_{k}\right\}$ of open subsets, where $I_{k}$ is some index set, and constants $C_{1}, C_{2}>0$ such that

(i) $\mu\left(X \backslash \cup_{\alpha} Q_{\alpha}^{k}\right)=0$ for each fixed $k$ and $Q_{\alpha}^{k} \cap Q_{\beta}^{k}=\emptyset$, if $\alpha \neq \beta$;

(ii) for any $\alpha, \beta, k, l$ with $l>k$, either $Q_{\beta}^{l} \subset Q_{\alpha}^{k}$ or $Q_{\beta}^{l} \cap$ $Q_{\alpha}^{k}=\emptyset$;

(iii) for each $(k, \alpha)$ and each $l<k$, there is a unique $\beta$ such that $Q_{\alpha}^{k} \subset Q_{\beta}^{l}$;

(iv) $\operatorname{diam}\left(Q_{\alpha}^{k}\right) \leq C_{1} 2^{-k}$;

(v) each $Q_{\alpha}^{k}$ contains some ball $B\left(y_{\alpha}^{k}, C_{2} 2^{-k}\right)$, where $y_{\alpha}^{k} \in$ $X$.

We think of $Q_{\alpha}^{k}$ as being a dyadic cube with diameter $2^{-k}$ and center $y_{\alpha}^{k}$. When $k \in \mathbb{Z}$ and $\tau \in I_{k}$, we denote by $Q_{\tau}^{k, v}$, where $v=1,2, \ldots, N(\tau, k)$, the set of all cubes $Q_{\tau}^{k+j} \subset Q_{\tau}^{k}$, where $j$ is a fixed large positive integer, and $y_{\tau}^{k, v}$ a point in $Q_{\tau}^{k, v}$.

Throughout the paper, we use $C$ to denote positive constants, whose value may change from one occurrence to the next. We denote by $f \sim g$ that there exists a constant $C>$ 0 independent of the main parameters such that $C^{-1} g<f<$ $\mathrm{Cg} \cdot \chi_{\mathrm{A}}$ is the characteristic function of $A$. Let $a_{+}=\max (0, a)$ for any $a \in \mathbb{R}$. We also denote $M_{1}, M_{2}$ are the HardyLittlewood maximal function on spaces of homogeneous type $X_{1}, X_{2}$, respectively.

We introduce Triebel-Lizorkin space $\dot{F}_{p}^{s, q}$ and Besov space $\dot{B}_{p}^{s, q}$ on product spaces of homogeneous type.

Definition 7. Suppose that $i=1,2, s=\left(s_{1}, s_{2}\right), s_{i} \in\left(-\epsilon_{i}, \epsilon_{i}\right)$ and let $\left\{D_{k_{i}}\right\}_{k_{i} \in \mathbb{Z}}$ be the same as in Lemma 5. Let $1<p, q<$ $\infty$ and $0<\beta_{i}, \gamma_{i}<\epsilon_{i}<\theta_{i}, f \in\left(\mathscr{G}^{\circ}\left(\beta_{1}, \beta_{2} ; \gamma_{1}, \gamma_{2}\right)\right)^{\prime}$. Then Triebel-Lizorkin space $\dot{F}_{p}^{s, q}\left(X_{1} \times X_{2}\right)$ is defined by

$$
\|f\|_{\dot{F}_{p}^{s, q}}:=\left\|\left(\sum_{k_{1}, k_{2} \in \mathbb{Z}} 2^{k_{1} s_{1} q} 2^{k_{2} s_{2} q}\left|D_{k_{1}} D_{k_{2}}(f)\right|^{q}\right)^{1 / q}\right\|_{p}<\infty .
$$

The Besov space $\dot{B}_{p}^{s, q}\left(X_{1} \times X_{2}\right)$ is defined by

$$
\|f\|_{\dot{B}_{p}^{s, q}}:=\left(\sum_{k_{1}, k_{2} \in \mathbb{Z}} 2^{k_{1} s_{1} q} 2^{k_{2} s_{2} q}\left\|D_{k_{1}} D_{k_{2}}(f)\right\|_{p}^{q}\right)^{1 / q}<\infty .
$$

In order to check that the definitions for $\dot{F}_{p}^{s, q}$ and $\dot{B}_{p}^{s, q}$ are independent of the choice of approximations to the identity, we recall almost orthogonality estimate.

Lemma 8 (see [15]). Let $i=1$,2. Suppose that $\left\{S_{k_{i}}\right\}_{k_{i} \in \mathbb{Z}}$ and $\left\{P_{k_{i}}\right\}_{k_{i} \in \mathbb{Z}}$ are two approximations to the identity on spaces of homogeneous type, and $D_{k_{i}}=S_{k_{i}}-S_{K_{i}-1}, E_{k_{i}}=P_{k_{i}}-P_{k_{i}-1}$. Then for $\epsilon_{i} \in\left(0, \theta_{i}\right)$, there exists a positive constant $C$ depending only on $\epsilon_{i}$ such that $D_{l_{1}} D_{l_{2}} E_{k_{1}} E_{k_{2}}\left(x_{1}, y_{1} ; x_{2}, y_{2}\right)$, the kernel of $D_{l_{1}} D_{l_{2}} E_{k_{1}} E_{k_{2}}$, satisfies the following estimate:

$$
\begin{aligned}
& \left|D_{l_{1}} D_{l_{2}} E_{k_{1}} E_{k_{2}}\left(x_{1}, y_{1} ; x_{2}, y_{2}\right)\right| \\
& \leq C 2^{-\left|k_{1}-l_{1}\right| \epsilon_{1}} 2^{-\left|k_{2}-l_{2}\right| \epsilon_{2}} \frac{2^{-\left(k_{1} \wedge l_{1}\right) \epsilon_{1}}}{\left(2^{-\left(k_{1} \wedge l_{1}\right)}+\rho_{1}\left(x_{1}, y_{1}\right)\right)^{1+\epsilon_{1}}} \\
& \quad \times \frac{2^{-\left(k_{2} \wedge l_{2}\right) \epsilon_{2}}}{\left(2^{-\left(k_{2} \wedge l_{2}\right)}+\rho_{2}\left(x_{2}, y_{2}\right)\right)^{1+\epsilon_{2}}} .
\end{aligned}
$$

By Calderón's reproducing formula and almost orthogonality estimate, we can get the following product-type Plancherel-Pôlya inequalities. The proofs of the following theorems are similar to Proposition 4.1 in [10]. Here we omit the details.

Theorem 9. Suppose that $D_{k}$ and $E_{k}$ are the same as in Lemma 8 for $k \in \mathbb{Z}$. Let $1<p, q<\infty$, and $\left|s_{i}\right|<\epsilon_{i}$ for $i=1,2$. 
Then there exists a constant $C>0$ such that for all $f \in$ $\left(\mathscr{G}^{\circ}\left(\beta_{1}, \beta_{2} ; \gamma_{1}, \gamma_{2}\right)\right)^{\prime}$ with $0<\beta_{i}, \gamma_{i}<\epsilon_{i}<\theta_{i}$ for $i=1,2$, and then

$$
\begin{gathered}
\left\|\left\{\sum_{k_{1} \in \mathbb{Z} k_{2} \in \mathbb{Z}}\left(2^{k_{1} s_{1}} 2^{k_{2} s_{2}}\left|D_{k_{1}} D_{k_{2}}(f)\right|\right)^{q}\right\}^{1 / q}\right\|_{L^{p}} \\
\sim\left\|\left\{\sum_{l_{1} \in \mathbb{Z} l_{2} \in \mathbb{Z}}\left(2^{l_{1} s_{1}} 2^{l_{2} s_{2}}\left|E_{l_{1}} E_{l_{2}}(f)\right|\right)^{q}\right\}^{1 / q}\right\|_{L^{p}}, \\
\left\{\sum_{k_{1} \in \mathbb{Z} k_{2} \in \mathbb{Z}}\left(2^{k_{1} s_{1}} 2^{k_{2} s_{2}}\left\|D_{k_{1}} D_{k_{2}}(f)\right\|_{L^{p}}\right)^{q}\right\}^{1 / q} \\
\sim\left\{\sum_{l_{1} \in \mathbb{Z} l_{2} \in \mathbb{Z}} \sum\left(2^{l_{1} s_{1}} 2^{l_{2} s_{2}}\left\|E_{l_{1}} E_{l_{2}}(f)\right\|_{L^{p}}\right)^{q}\right\}^{1 / q} .
\end{gathered}
$$

Remark 10. Let $D_{l_{1}}, D_{l_{2}}, \bar{D}_{k_{1}}$, and $\bar{D}_{k_{2}}$ be as in Lemma 5. Then the kernel of $D_{l_{1}} D_{l_{2}}$ has compact support, but not of $\bar{D}_{k_{1}} \bar{D}_{k_{2}}$. Since (11) holds for $D_{l_{1}}$ and $D_{l_{2}}$ satisfying only the smoothness condition for the second variable, we conclude that Theorem 9 holds with $D_{l_{1}} D_{l_{2}}$ being replaced by $\bar{D}_{k_{1}} \bar{D}_{k_{2}}$.

\section{Smooth Atomic Decomposition}

In this section, smooth atomic decomposition is presented. We first give definitions of the smooth atoms for $\dot{B}_{p}^{s, q}$ and $\dot{F}_{p}^{s, q}$.

Definition 11. Suppose collections of open subset $\left\{Q_{\tau_{1}}^{k_{1}, v_{1}} \subset\right.$ $\left.X_{1}: k_{1} \in \mathbb{Z}, \tau_{1} \in I_{k_{1}}, v_{1}=1, \ldots, N\left(k_{1}, \tau_{1}\right)\right\}$ and $\left\{Q_{\tau_{2}}^{k_{2}, v_{2}} \subset\right.$ $\left.X_{2}: k_{2} \in \mathbb{Z}, \tau_{2} \in I_{k_{2}}, v_{2}=1, \ldots, N\left(k_{2}, \tau_{2}\right)\right\}$ satisfying the conditions in Lemma 6. Let $\epsilon=\left(\epsilon_{1}, \epsilon_{2}\right)$ and $R=Q_{\tau_{1}}^{k_{1}, v_{1}} \times Q_{\tau_{2}}^{k_{2}, v_{2}}$, and a function $a_{R}$ defined on $X_{1} \times X_{2}$ is said to be a $\epsilon$-smooth atom for $R$ if

(i)

$$
\operatorname{supp} a_{R} \subset B_{1}\left(z_{\tau_{1}}^{k_{1}, v_{1}}, A_{1} C 2^{-k_{1}}\right) \times B_{2}\left(z_{\tau_{2}}^{k_{2}, v_{2}}, A_{2} C 2^{-k_{2}}\right) \text {, }
$$

where $z_{\tau_{i}}^{k_{i}, v_{i}}$ is the center of $Q_{\tau_{i}}^{k_{i}, v_{i}}$ for $i=1,2$;

(ii)

$$
\int_{X_{2}} a_{R}\left(x_{1}, x_{2}\right) d \mu_{2}\left(x_{2}\right)=\int_{X_{1}} a_{R}\left(x_{1}, x_{2}\right) d \mu_{1}\left(x_{1}\right)=0 ;
$$

(iii)

$$
\begin{aligned}
& \left|a_{R}(x, y)\right| \leq C \mu_{1}\left(Q_{\tau_{1}}^{k_{1}, v_{1}}\right)^{-1 / 2} \mu_{2}\left(Q_{\tau_{2}}^{k_{2}, v_{2}}\right)^{-1 / 2}, \\
& \left|a_{R}(x, y)-a_{R}\left(x^{\prime}, y\right)\right| \\
& \leq C \mu_{1}\left(Q_{\tau_{1}}^{k_{1}, v_{1}}\right)^{-1 / 2-\epsilon_{1}} \mu_{2}\left(Q_{\tau_{2}}^{k_{2}, v_{2}}\right)^{-1 / 2} \rho_{1}\left(x, x^{\prime}\right)^{\epsilon_{1}},
\end{aligned}
$$

$$
\begin{aligned}
& \left|a_{R}(x, y)-a_{R}\left(x, y^{\prime}\right)\right| \\
& \leq C \mu_{1}\left(Q_{\tau_{1}}^{k_{1}, v_{1}}\right)^{-1 / 2} \mu_{2}\left(Q_{\tau_{2}}^{k_{2}, v_{2}}\right)^{-1 / 2-\epsilon_{2}} \rho_{2}\left(y, y^{\prime}\right)^{\epsilon_{2}}, \\
& \left|a_{R}(x, y)-a_{R}\left(x^{\prime}, y\right)-a_{R}\left(x, y^{\prime}\right)-a_{R}\left(x^{\prime}, y^{\prime}\right)\right| \\
& \leq C \mu_{1}\left(Q_{\tau_{1}}^{k_{1}, v_{1}}\right)^{-1 / 2-\epsilon_{1}} \mu_{2}\left(Q_{\tau_{2}}^{k_{2}, v_{2}}\right)^{-1 / 2-\epsilon_{2}} \\
& \quad \times \rho_{1}\left(x, x^{\prime}\right)^{\epsilon_{1}} \rho_{2}\left(y, y^{\prime}\right)^{\epsilon_{2}} .
\end{aligned}
$$

Now, we also define certain spaces of sequences of indexed by dyadic rectangles $\left\{Q_{\tau_{1}}^{k_{1}, v_{1}} \times Q_{\tau_{2}}^{k_{2}, v_{2}}\right\}$ in $X_{1} \times X_{2}$ which will character the coefficients in our decomposition of $\dot{F}_{p}^{s, q}$ and $\dot{B}_{p}^{s, q}$. Suppose that $s=\left(s_{1}, s_{2}\right), \epsilon=\left(\epsilon_{1}, \epsilon_{2}\right)$ with $-\epsilon_{i}<s_{i}<\epsilon_{i}$ for $i=1,2,1<p, q<\infty$. Let $R$ be as in Definition 11. Let $\dot{f}_{p}^{s, q}$ and $\dot{b}_{p}^{s, q}$ be the collection of sequences $S=\left\{s_{\mathrm{Q}_{1} \times \mathrm{Q}_{2}}\right\}_{\mathrm{Q}_{1} \times \mathrm{Q}_{2} \in\{R\}}$ such that

$\|S\|_{f_{p}^{s, q}}$

$$
\begin{aligned}
=\|\left\{\sum_{k_{1}, k_{2}} \sum_{\tau_{1}, \tau_{2}} \sum_{v_{1}, v_{2}}(\right. & \mu_{1}\left(Q_{\tau_{1}}^{k_{1}, v_{1}}\right)^{-s_{1}-1 / 2} \\
& \left.\left.\times \mu_{2}\left(Q_{\tau_{2}}^{k_{2}, v_{2}}\right)^{-s_{2}-1 / 2}\left|s_{R}\right| \chi_{R}\right)^{q}\right\}^{1 / q} \|_{L^{p}}
\end{aligned}
$$

$<\infty$,

$\|S\|_{\dot{b}_{p}^{s, q}}$

$$
\begin{aligned}
&=\left\{\sum _ { k _ { 1 } , k _ { 2 } } \left(\sum_{\tau_{1}, \tau_{2} \nu_{1}, v_{2}}(\right.\right.\left(\mu_{1}\left(Q_{\tau_{1}}^{k_{1}, v_{1}}\right)^{-s_{1}}\right. \\
&\left.\left.\left.\left.\times \mu_{2}\left(Q_{\tau_{2}}^{k_{2}, v_{2}}\right)^{-s_{2}}\right)^{-1 / 2+1 / p}\left|s_{R}\right|\right)^{p}\right)^{q / p}\right\}^{1 / q}
\end{aligned}
$$

$<\infty$.

Smooth atomic decompositions for $\dot{B}_{p}^{s, q}$ and $\dot{F}_{p}^{s, q}$ can be stated as follows.

Theorem 12. Let $R$ be as in Definition 11. Suppose that $1<p$, $q<\infty, s=\left(s_{1}, s_{2}\right), \epsilon=\left(\epsilon_{1}, \epsilon_{2}\right)$ with $-\epsilon_{i}<s_{i}<\epsilon_{i}$ for $i=1,2$.

(i) Then there exist sequence $S=\left\{s_{R}\right\} \in \dot{b}_{p}^{s, q}$ and $\varepsilon$-smooth atoms $\left\{a_{R}\right\}$ such that

$$
f=\sum_{k_{1}, k_{2} \in \mathbb{Z}} \sum_{\tau_{1} \in I_{k_{1}}} \sum_{v_{1}=1}^{N\left(k_{1}, \tau_{1}\right)} \sum_{\tau_{2} \in I_{k_{2}}} \sum_{v_{2}=1}^{N\left(k_{2}, \tau_{2}\right)} s_{R} a_{R}
$$

with convergence in $\dot{B}_{p}^{s, q}$, and

$$
\|S\|_{\dot{b}_{p}^{s, q}} \leq C\|f\|_{\dot{B}_{p}^{s, q} .}
$$


Similarly, there exist sequence $S=\left\{s_{R}\right\} \in \dot{f}_{p}^{s, q}$ and $\epsilon$ smooth atoms $\left\{a_{R}\right\}$ such that

$$
f=\sum_{k_{1}, k_{2} \in \mathbb{Z}} \sum_{\tau_{1} \in I_{k_{1}}} \sum_{v_{1}=1}^{N\left(k_{1}, \tau_{1}\right)} \sum_{\tau_{2} \in I_{k_{2}}} \sum_{v_{2}=1}^{N\left(k_{2}, \tau_{2}\right)} s_{R} a_{R}
$$

with convergence in $\dot{F}_{p}^{s, q}$ and

$$
\|S\|_{\dot{f}_{p}^{s, q}} \leq C\|f\|_{\dot{F}_{p}^{s, q}}
$$

(ii) Conversely, if $a_{R}$ are $\epsilon$-smooth atoms and

$$
f=\sum_{k_{1}, k_{2} \in \mathbb{Z}} \sum_{\tau_{1} \in I_{k_{1}}} \sum_{v_{1}=1}^{N\left(k_{1}, \tau_{1}\right)} \sum_{\tau_{2} \in I_{k_{2}}} \sum_{v_{2}=1}^{N\left(k_{2}, \tau_{2}\right)} s_{R} a_{R}
$$

then

$$
\|f\|_{\dot{B}_{p}^{s, q}} \leq C\|S\|_{\dot{b}_{p}^{s, q}}, \quad\|f\|_{\dot{F}_{p}^{s, q}} \leq C\|S\|_{f_{p}^{s, q}}
$$

Proof. We first prove part (i). Let $R$ be as in Definition 11 . Suppose $f \in \dot{B}_{p}^{s, q}$; by Lemma 5, we have

$$
\begin{aligned}
f(x, y)= & \sum_{k_{1}, k_{2} \in \mathbb{Z}} \sum_{\tau_{1} \in I_{k_{1}}} \sum_{v_{1}=1}^{N\left(k_{1}, \tau_{1}\right)} \sum_{\tau_{2} \in I_{k_{2}}} \sum_{v_{2}=1}^{N\left(k_{2}, \tau_{2}\right)} \int_{R} D_{k_{1}}(x, s) \\
& \times D_{k_{2}}(t, y) \bar{D}_{k_{1}} \bar{D}_{k_{2}}(f)(s, t) d \mu_{1}(s) d \mu_{2}(t) \\
:= & \sum_{k_{1}, k_{2} \in \mathbb{Z}} \sum_{\tau_{1} \in I_{k_{1}}} \sum_{v_{1}=1}^{N\left(k_{1}, \tau_{1}\right)} \sum_{\tau_{2} \in I_{k_{2}}} \sum_{v_{2}=1}^{N\left(k_{2}, \tau_{2}\right)} s_{R} a_{R},
\end{aligned}
$$

where

$$
\begin{aligned}
s_{R}= & C \mu_{1}\left(Q_{\tau_{1}}^{k_{1}, v_{1}}\right)^{-1 / 2} \mu_{2}\left(Q_{\tau_{2}}^{k_{2}, v_{2}}\right)^{-1 / 2} \\
& \times \int_{R}\left|\bar{D}_{k_{1}} \bar{D}_{k_{2}}(f)(s, t)\right| d \mu_{1}(s) d \mu_{2}(t), \\
a_{R}= & C^{-1} \mu_{1}\left(Q_{\tau_{1}}^{k_{1}, v_{1}}\right)^{1 / 2} \mu_{2}\left(Q_{\tau_{2}}^{k_{2}, v_{2}}\right)^{1 / 2} \\
& \times\left(\int_{R}\left|\bar{D}_{k_{1}} \bar{D}_{k_{2}}(f)(s, t)\right| d \mu_{1}(s) d \mu_{2}(t)\right)^{-1} \\
& \times \int_{R} D_{k_{1}}(x, s) D_{k_{2}}(t, y) \bar{D}_{k_{1}} \bar{D}_{k_{2}}(f)(s, t) \\
& \times d \mu_{1}(s) d \mu_{2}(t) .
\end{aligned}
$$

Similar to $[7,10]$, we can prove the convergence of the above series converges in $\dot{B}_{p}^{s, q}$ and $\left(\mathscr{G}^{\circ}\left(\beta_{1}^{\prime}, \beta_{2}^{\prime}, \gamma_{1}^{\prime}, \gamma_{2}^{\prime}\right)\right)^{\prime}$ with $\beta_{i}<\beta_{i}^{\prime}<$ $\epsilon_{i}$ and $\gamma_{i}<\gamma_{i}^{\prime}<\epsilon_{i}$ for $i=1,2$. We now show that $a_{R}$ satisfies conditions in Definition 11 . Here we only show that $a_{R}$ satisfies condition (iii) and we first prove that $a_{R}$ satisfies
(15). By the size condition of $D_{k_{i}}$ and $\mu_{i}\left(Q_{\tau_{i}}^{k_{i}, v_{i}}\right) \sim 2^{-k_{i}}$ for $i=1,2$, we have

$$
\begin{aligned}
& \left|a_{R}(x, y)\right| \\
& \leq C \mu_{1}\left(Q_{\tau_{1}}^{k_{1}, v_{1}}\right)^{1 / 2} \mu_{2}\left(Q_{\tau_{2}}^{k_{2}, v_{2}}\right)^{1 / 2} \\
& \quad \times\left(\int_{R}\left|\bar{D}_{k_{1}} \bar{D}_{k_{2}}(f)(s, t)\right| d \mu_{1}(s) d \mu_{2}(t)\right)^{-1} \\
& \quad \times \int_{R} \frac{2^{-k_{1} \epsilon_{1}}}{\left(2^{-k_{1}}+\rho_{1}(x, s)\right)^{1+\epsilon_{1} \epsilon_{2}}} \frac{\left.2^{-k_{2}}+\rho_{2}(y, t)\right)^{1+\epsilon_{2}}}{\left(2^{2}\right.} \\
& \quad \times\left|\bar{D}_{k_{1}} \bar{D}_{k_{2}}(f)(s, t)\right| d \mu_{1}(s) d \mu_{2}(t) \\
& \leq C \mu_{1}\left(Q_{\tau_{1}}^{k_{1}, v_{1}}\right)^{1 / 2} \mu_{2}\left(Q_{\tau_{2}}^{k_{2}, v_{2}}\right)^{1 / 2} 2^{k_{1}} 2^{k_{2}} \\
& \leq C \mu_{1}\left(Q_{\tau_{1}}^{k_{1}, v_{1}}\right)^{-1 / 2} \mu_{2}\left(Q_{\tau_{2}}^{k_{2}, v_{2}}\right)^{-1 / 2} .
\end{aligned}
$$

We now verify (16); here, we only consider the case $\rho_{1}\left(x, x^{\prime}\right) \leq(1 / 2 A)\left(2^{-k_{1}}+\rho_{1}(x, s)\right)$ and similarly we estimate the case $\rho_{1}\left(x, x^{\prime}\right)>(1 / 2 A)\left(2^{-k_{1}}+\rho_{1}(x, s)\right)$; we get

$$
\begin{aligned}
\mid a_{R}( & x, y)-a_{R}\left(x^{\prime}, y\right) \mid \\
\leq & C \mu_{1}\left(Q_{\tau_{1}}^{k_{1}, v_{1}}\right)^{1 / 2} \mu_{2}\left(Q_{\tau_{2}}^{k_{2}, v_{2}}\right)^{1 / 2} \\
\times & \left(\int_{R}\left|\bar{D}_{k_{1}} \bar{D}_{k_{2}}(f)(s, t)\right| d \mu_{1}(s) d \mu_{2}(t)\right)^{-1} \\
\times & \int_{R}\left|D_{k_{1}}(x, s)-D_{k_{1}}\left(x^{\prime}, s\right)\right|\left|D_{k_{2}}(t, y)\right| \\
\leq & C \mu_{1}\left(Q_{\tau_{1}}^{k_{1}, v_{1}}\right)^{1 / 2} \mu_{2}\left(Q_{\tau_{2}}^{k_{2}, v_{2}}\right)^{1 / 2} \\
\times & \left(\int_{R}\left|\bar{D}_{k_{1}} \bar{D}_{k_{2}}(f)(s, t)\right| d \mu_{1}(s) d \mu_{2}(t)\right)^{-1} \\
\times & \quad \int_{R}\left(\frac{\rho_{1}\left(x, x^{\prime}\right)}{2^{-k_{1}}+\rho_{1}(x, s)}\right)^{\epsilon_{1}} \\
& \times\left|\bar{D}_{k_{1}} \bar{D}_{k_{2}}(f)(s, t)\right| d \mu_{1}(s) d \mu_{2}(t) \\
& \times \frac{2^{-k_{1} \epsilon_{1}}}{\left.\left(2_{\tau_{1}}^{k_{1}, v_{1}}\right)^{-1 / 2-\epsilon_{1}}+\mu_{1}(x, s)\right)^{1+\epsilon_{1}}} \\
& \times \frac{2 \mu^{-k_{2} \epsilon_{2}}}{\left(2^{-k_{2}}+\rho_{2}(y, t)\right)^{1+\epsilon_{2}}} \\
& \left.\quad \rho_{\tau_{2}, v_{2}}\right)^{-1 / 2} \rho_{1}\left(x, x^{\prime}\right)^{\epsilon_{1}},
\end{aligned}
$$

which is a desired estimate. The proof of (17) is similar to that of (16) by symmetry. Here we omit the details. 
We now estimate (18). If $\rho_{1}\left(x, x^{\prime}\right) \leq(1 / 2 A)\left(2^{-k_{1}}+\rho_{1}(x, s)\right)$ and $\rho_{2}\left(y, y^{\prime}\right) \leq(1 / 2 A)\left(2^{-k_{2}}+\rho_{2}(y, t)\right)$, then

$$
\begin{aligned}
& \left|a_{R}(x, y)-a_{R}\left(x^{\prime}, y\right)-a_{R}\left(x, y^{\prime}\right)-a_{R}\left(x^{\prime}, y^{\prime}\right)\right| \\
& \leq C \mu_{1}\left(Q_{\tau_{1}}^{k_{1}, v_{1}}\right)^{1 / 2} \mu_{2}\left(Q_{\tau_{2}}^{k_{2}, v_{2}}\right)^{1 / 2} \\
& \times\left(\int_{R}\left|\bar{D}_{k_{1}} \bar{D}_{k_{2}}(f)(s, t)\right| d \mu_{1}(s) d \mu_{2}(t)\right)^{-1} \\
& \times \int_{R}\left|D_{k_{1}}(x, s)-D_{k_{1}}\left(x^{\prime}, s\right)\right| \\
& \times\left|D_{k_{2}}(t, y)-D_{k_{2}}\left(t, y^{\prime}\right)\right| \\
& \times\left|\bar{D}_{k_{1}} \bar{D}_{k_{2}}(f)(s, t)\right| d \mu_{1}(s) d \mu_{2}(t) \\
& \leq C \mu_{1}\left(Q_{\tau_{1}}^{k_{1}, v_{1}}\right)^{1 / 2} \mu_{2}\left(Q_{\tau_{2}}^{k_{2}, v_{2}}\right)^{1 / 2} \\
& \times\left(\int_{R}\left|\bar{D}_{k_{1}} \bar{D}_{k_{2}}(f)(s, t)\right| d \mu_{1}(s) d \mu_{2}(t)\right)^{-1} \\
& \times \int_{R}\left(\frac{\rho_{1}\left(x, x^{\prime}\right)}{2^{-k_{1}}+\rho_{1}(x, s)}\right)^{\epsilon_{1}}\left(\frac{\rho_{2}\left(y, y^{\prime}\right)}{2^{-k_{2}}+\rho_{2}(y, t)}\right)^{\epsilon_{2}} \\
& \times \frac{2^{-k_{1} \epsilon_{1}}}{\left(2^{-k_{1}}+\rho_{1}(x, s)\right)^{1+\epsilon_{1}}} \\
& \times \frac{2^{-k_{2} \epsilon_{2}}}{\left(2^{-k_{2}}+\rho_{2}(y, t)\right)^{1+\epsilon_{2}}} \\
& \times\left|\bar{D}_{k_{1}} \bar{D}_{k_{2}}(f)(s, t)\right| d \mu_{1}(s) d \mu_{2}(t) \\
& \leq C \mu_{1}\left(Q_{\tau_{1}}^{k_{1}, v_{1}}\right)^{-1 / 2-\epsilon_{1}} \mu_{2}\left(Q_{\tau_{2}}^{k_{2}, v_{2}}\right)^{-1 / 2-\epsilon_{2}} \\
& \times \rho_{1}\left(x, x^{\prime}\right)^{\epsilon_{1}} \rho_{2}\left(y, y^{\prime}\right)^{\epsilon_{2}},
\end{aligned}
$$

which is the desired result. Similarly we can consider another three cases.

Then, by Hölder's inequality and Remark 10, we obtain

$\|S\|_{b_{p}^{s, q}}$

$$
\begin{aligned}
=\left\{\sum_{k_{1}, k_{2} \in \mathbb{Z}}\right. & \left(\sum_{\tau_{1} \in I_{k_{1}}} \sum_{v_{1}=1}^{N\left(k_{1}, \tau_{1}\right)} \sum_{\tau_{2} \in I_{k_{2}}} \sum_{v_{2}=1}^{N\left(k_{2}, \tau_{2}\right)}\right. \\
& \left(\mu_{1}\left(Q_{\tau_{1}}^{k_{1}, v_{1}}\right)^{-s_{1}-1 / 2+1 / p} \mu_{2}\left(Q_{\tau_{2}}^{k_{2}, v_{2}}\right)^{-s_{2}-1 / 2+1 / p}\right. \\
& \times C \mu_{1}\left(Q_{\tau_{1}}^{k_{1}, v_{1}}\right)^{-1 / 2} \mu_{2}\left(Q_{\tau_{2}}^{k_{2}, v_{2}}\right)^{-1 / 2} \\
& \left.\left.\left.\times \int_{R}\left|\bar{D}_{k_{1}} \bar{D}_{k_{2}}(f)(s, t)\right| d \mu_{1}(s) d \mu_{2}(t)\right)^{p}\right)^{q / p}\right\}^{1 / q}
\end{aligned}
$$

$\leq C\left\{\sum_{k_{1}, k_{2} \in \mathbb{Z}}\left(\sum_{\tau_{1} \in I_{k_{1}}} \sum_{v_{1}=1}^{N\left(k_{1}, \tau_{1}\right)} \sum_{\tau_{2} \in I_{k_{2}}} \sum_{v_{2}=1}^{N\left(k_{2}, \tau_{2}\right)} 2^{k_{1} s_{1} p} 2^{k_{2} s_{2} p}\right.\right.$

$$
\begin{aligned}
& \times \mu_{1}\left(Q_{\tau_{1}}^{k_{1}, v_{1}}\right)^{-p / p^{\prime}} \mu_{2}\left(Q_{\tau_{2}}^{k_{2}, v_{2}}\right)^{-p / p^{\prime}} \\
& \times\left(\int_{R} \bar{D}_{k_{1}} \bar{D}_{k_{2}}(f)(s, t)\right.
\end{aligned}
$$$$
\left.\left.\left.\times d \mu_{1}(s) d \mu_{2}(t)\right)^{p}\right)^{q / p}\right\}^{1 / q}
$$$$
\leq C\left\{\sum_{k_{1}, k_{2} \in \mathbb{Z}}\left(2^{k_{1} s_{1}} 2^{k_{2} s_{2}}\left\|\bar{D}_{k_{1}} \bar{D}_{k_{2}}(f)\right\|_{p}\right)^{q}\right\}^{1 / q} \leq C\|f\|_{\dot{B}_{p}^{s, q} .} .
$$

The proof of the case $f \in \dot{F}_{p}^{s, q}$ goes by the analogous argument to the case that $f \in \dot{B}_{p}^{s, q}$, but using the vectorvalued maximal inequality. Indeed, suppose that $f \in \dot{F}_{p}^{s, q}$; as in the proof of $\dot{B}_{p}^{s, q}$, we have

$$
f=\sum_{k_{1}, k_{2} \in \mathbb{Z}} \sum_{\tau_{1} \in I_{k_{1}}} \sum_{v_{1}=1}^{N\left(k_{1}, \tau_{1}\right)} \sum_{\tau_{2} \in I_{k_{2}}} \sum_{v_{2}=1}^{N\left(k_{2}, \tau_{2}\right)} s_{R} a_{R}
$$

where $s_{R}$ and $a_{R}$ are as in (27) and (28), respectively. From $[7,10]$, we can also similarly obtain the series converges in the norm of $\dot{F}_{p}^{s, q}$ and in $\left(\mathscr{G}^{\circ}\left(\beta_{1}^{\prime}, \beta_{2}^{\prime}, \gamma_{1}^{\prime}, \gamma_{2}^{\prime}\right)\right)^{\prime}$ with $\beta_{i}<\beta_{i}^{\prime}<\epsilon_{i}$ and $\gamma_{i}<\gamma_{i}^{\prime}<\epsilon_{i}$ for $i=1,2$. To show the conclusion, using Fefferman-Stein vector-valued maximal inequality and Remark 10, we have

$\|S\|_{f_{p}^{s, q}}$

$$
\begin{aligned}
& =\|\left\{\sum _ { k _ { 1 } , k _ { 2 } \in \mathbb { Z } } \sum _ { \tau _ { 1 } \in I _ { k _ { 1 } } } \sum _ { v _ { 1 } = 1 } ^ { N ( k _ { 1 } , \tau _ { 1 } ) } \sum _ { \tau _ { 2 } \in I _ { k _ { 2 } } } \sum _ { v _ { 2 } = 1 } ^ { N ( k _ { 2 } , \tau _ { 2 } ) } \left(\mu_{1}\left(Q_{\tau_{1}}^{k_{1}, v_{1}}\right)^{-s_{1}-1 / 2}\right.\right. \\
& \quad \times \mu_{2}\left(Q_{\tau_{2}}^{k_{2}, v_{2}}\right)^{-s_{2}-1 / 2} C \mu_{1}\left(Q_{\tau_{1}}^{k_{1}, v_{1}}\right)^{-1 / 2} \mu_{2}\left(Q_{\tau_{2}}^{k_{2}, v_{2}}\right)^{-1 / 2}
\end{aligned}
$$

$$
\begin{aligned}
& \left.\left.\times \int_{R}\left|\bar{D}_{k_{1}} \bar{D}_{k_{2}}(f)(s, t)\right| d \mu_{1}(s) d \mu_{2}(t) \chi_{\mathrm{Q}_{\tau_{1}}^{k_{1}, v_{1}}} \chi_{\mathrm{Q}_{\tau_{2}}^{k_{2}, v_{2}}}\right)^{q}\right\}^{1 / q} \|_{p} \\
\leq & C\left\|\left\{\sum_{k_{1}, k_{2} \in \mathbb{Z}}\left(2^{k_{1} s_{1}} 2^{k_{2} s_{2}} M_{2}\left(M_{1}\left(\bar{D}_{k_{1}} \bar{D}_{k_{2}}(f)\right)\right)\right)^{q}\right\}^{1 / q}\right\|_{p} \\
\leq & C\left\|\left\{\sum_{k_{1}, k_{2} \in \mathbb{Z}}\left(2^{k_{1} s_{1}} 2^{k_{2} s_{2}} \bar{D}_{k_{1}} \bar{D}_{k_{2}}(f)\right)^{q}\right\}^{1 / q}\right\|_{p} \leq C\|f\|_{\dot{F}_{p}^{s, q}} .
\end{aligned}
$$


To prove part (ii), we need the discrete version of the Hardy-Littlewood maximal function estimate on one single factor $X$, which is an analogue to a result of [9]. Also see [10].

Lemma 13. Suppose that $X$ is a space of homogeneous type as in Definition 1 Let $0<a \leq r<\infty$ and $\lambda>r / a$. Fix $\mu, \eta$ with $\mu, \eta \in \mathbb{Z}$ and for any $x \in X$

$$
\begin{gathered}
\left(\sum_{\tau \in I_{\mu}} \sum_{v=1}^{N(\mu, \tau)}\left|s_{Q_{\tau}^{\mu \nu}}\right|^{r}\left(1+\min \left\{2^{\eta}, 2^{\mu}\right\} \rho\left(x, z_{\tau}^{\mu, \nu}\right)\right)^{-\lambda}\right)^{1 / r} \\
\leq C 2^{(\mu-\eta)_{+} / a}\left(M\left(\sum_{\tau \in I_{\mu}} \sum_{v=1}^{N(\mu, \tau)}\left|s_{Q_{\tau}, \nu}\right|^{a} \chi_{Q_{\tau}^{\mu, \nu}}\right)(x)\right)^{1 / a},
\end{gathered}
$$

where $z_{\tau}^{\mu, v}$ is the center of dyadic cube $Q_{\tau}^{\mu, v}$ and C depends only on $\lambda, r, a$.

We now turn to proof of part (ii). In this part, we denote $R=Q_{\tau_{1}}^{\mu_{1}, v_{1}} \times Q_{\tau_{2}}^{\mu_{2}, v_{2}}$. Suppose that $\left\{D_{k_{i}}\right\}_{k_{i} \in \mathbb{Z}}$ for $i=1,2$ is as in Lemma 5 and $a_{R}$ is an $\varepsilon$-smooth atom. For $D_{k_{1}} D_{k_{2}}\left(a_{R}\right)(x, y)$, we consider it by four cases. We first estimate the case $k_{1}>$ $\mu_{1}, k_{2}>\mu_{2}$. In this case, applying the cancellation conditions of $D_{k_{1}}$ and $D_{k_{2}}$,

$$
\begin{aligned}
& \left|D_{k_{1}} D_{k_{2}}\left(a_{R}\right)(x, y)\right| \\
& =\mid \int_{X_{1} \times X_{2}} D_{k_{1}}(x, s) D_{k_{2}}(y, t) \\
& \times\left[a_{R}(s, t)-a_{R}(x, t)-a_{R}(s, y)+a_{R}(x, y)\right] \\
& \times d \mu_{1}(s) d \mu_{2}(t) \\
& \leq C \mu_{1}\left(Q_{\tau_{1}}^{\mu_{1}, v_{1}}\right)^{-1 / 2-\epsilon_{1}} \mu_{2}\left(Q_{\tau_{2}}^{\mu_{1}, v_{2}}\right)^{-1 / 2-\epsilon_{2}} \\
& \times \int_{\substack{\rho_{1}(s, x) \leq 2 C 2^{-k_{1}} \\
\rho_{2}(t, y) \leq 2 \mathrm{C}^{-k_{2}}}} \frac{2^{-k_{1} \epsilon_{1}}}{\left(2^{-k_{1}}+\rho_{1}(x, s)\right)^{1+\epsilon_{1}}} \\
& \times \frac{2^{-k_{2} \epsilon_{2}}}{\left(2^{-k_{2}}+\rho_{2}(t, y)\right)^{1+\epsilon_{2}}} \rho_{1}(x, s)^{\epsilon_{1}} \\
& \times \rho_{2}(t, y)^{\epsilon_{2}} d \mu_{1}(s) d \mu_{2}(t) \\
& \leq C \mu_{1}\left(Q_{\tau_{1}}^{\mu_{1}, v_{1}}\right)^{-1 / 2-\epsilon_{1}} \mu_{2}\left(Q_{\tau_{2}}^{\mu_{2}, v_{2}}\right)^{-1 / 2-\epsilon_{2}} \\
& \times \int_{\substack{\rho_{1}(s, x) \leq 2 C 2^{-k_{1}} \\
\rho_{2}(t, y) \leq 2 C 2^{-k_{2}}}} d \mu_{1}(s) d \mu_{2}(t) \\
& \times \frac{2^{k_{1}} 2^{-k_{1} \epsilon_{1}}}{\left(1+2^{\mu_{1}} \rho_{1}\left(x, z_{\tau_{1}}^{\mu_{1}, v_{1}}\right)\right)^{1+\epsilon_{1}}} \\
& \times \frac{2^{k_{2}} 2^{-k_{2} \epsilon_{2}}}{\left(1+2^{\mu_{2}} \rho_{2}\left(y, z_{\tau_{2}}^{\mu_{2}, v_{2}}\right)\right)^{1+\epsilon_{2}}}
\end{aligned}
$$

$$
\begin{aligned}
\leq & C \mu_{1}\left(Q_{\tau_{1}}^{\mu_{1}, v_{1}}\right)^{-1 / 2} \mu_{2}\left(Q_{\tau_{2}}^{\mu_{2}, v_{2}}\right)^{-1 / 2} \\
& \times \frac{2^{-\left(k_{1}-\mu_{1}\right) \epsilon_{1}}}{\left(1+2^{\mu_{1}} \rho_{1}\left(x, z_{\tau_{1}}^{\mu_{1}, v_{1}}\right)\right)^{1+\epsilon_{1}}} \\
& \times \frac{2^{-\left(k_{2}-\mu_{2}\right) \epsilon_{2}}}{\left(1+2^{\mu_{2}} \rho_{2}\left(x, z_{\tau_{2}}^{\mu_{2}, v_{2}}\right)\right)^{1+\epsilon_{2}}} .
\end{aligned}
$$

To estimate the case $k_{1}>\mu_{1}, k_{2} \leq \mu_{2}$, we only estimate $\rho_{2}\left(t, z_{\tau_{2}}^{u_{2}, v_{2}}\right) \leq(1 / 2 A)\left(2^{-k_{2}}+\rho_{2}(y, t)\right)$, and afterwards we can consider the case $\rho_{2}\left(t, z_{\tau_{2}}^{u_{2}, v_{2}}\right)>(1 / 2 A)\left(2^{-k_{2}}+\rho_{2}(y, t)\right)$ similarly. In this case, we have

$$
\begin{aligned}
& \left|D_{k_{1}} D_{k_{2}}\left(a_{R}\right)(x, y)\right| \\
& =\mid \int_{X_{1} \times X_{2}} D_{k_{1}}(x, s)\left[D_{k_{2}}(y, t)-D_{k_{2}}\left(y, z_{\tau_{2}}^{u_{2}, v_{2}}\right)\right] \\
& \times\left[a_{R}(s, t)-a_{R}(x, t)\right] d \mu_{1}(s) d \mu_{2}(t) \\
& \leq C \mu_{1}\left(Q_{\tau_{1}}^{\mu_{1}, v_{1}}\right)^{-1 / 2-\epsilon_{1}} \mu_{2}\left(Q_{\tau_{2}}^{\mu_{2}, v_{2}}\right)^{-1 / 2} \\
& \times \int_{\substack{\rho_{1}(s, x) \leq 2 C^{-k_{1}} \\
\rho_{2}\left(t, z_{\tau_{2}, v_{2}}^{u_{2}}\right) \leq 2 C 2^{-\mu_{2}}}} \frac{2^{-k_{1} \epsilon_{1}}}{\left(2^{-k_{1}}+\rho_{1}(x, s)\right)^{1+\epsilon_{1}}} \\
& \times\left(\frac{\rho_{2}\left(t, z_{\tau_{2}}^{u_{2}, v_{2}}\right)}{2^{-k_{2}}+\rho_{2}(t, y)}\right)^{\epsilon_{2}} \frac{2^{-k_{2} \epsilon_{2}}}{\left(2^{-k_{2}}+\rho_{2}(t, y)\right)^{1+\epsilon_{2}}} \\
& \times \rho_{1}(x, s)^{\epsilon_{1}} d \mu_{1}(s) d \mu_{2}(t) \\
& \leq C \mu_{1}\left(Q_{\tau_{1}}^{\mu_{1}, v_{1}}\right)^{-1 / 2-\epsilon_{1}} \mu_{2}\left(Q_{\tau_{2}}^{\mu_{2}, v_{2}}\right)^{-1 / 2} \\
& \times \int_{\substack{\rho_{1}(s, x) \leq 2 C 2^{-k_{1}} \\
\rho_{2}\left(t, z_{\tau_{2}}, v_{2} \\
\tau_{2}\right.} \leq 2 C^{-\mu_{2}}} \frac{2^{k_{1}} 2^{-k_{1} \epsilon_{1}}}{\left(1+2^{\mu_{1}} \rho_{1}\left(x, z_{\tau_{1}}^{\mu_{1}, v_{1}}\right)\right)^{1+\epsilon_{1}}} \\
& \times \frac{2^{-\mu_{2} \epsilon_{2}} 2^{k_{2}} 2^{k_{2} \epsilon_{2}}}{\left(1+2^{k_{2}} \rho_{2}\left(y, z_{\tau_{2}}^{\mu_{2}, v_{2}}\right)\right)^{1+\epsilon_{2}}} d \mu_{1}(s) d \mu_{2}(t) \\
& \leq C \mu_{1}\left(Q_{\tau_{1}}^{\mu_{1}, v_{1}}\right)^{-1 / 2-\epsilon_{1}} \mu_{2}\left(Q_{\tau_{2}}^{\mu_{2}, v_{2}}\right)^{-1 / 2} \\
& \times \int_{\substack{\rho_{1}(s, x) \leq 2 C 2^{-k_{1}} \\
\rho_{2}\left(t, z_{\tau_{2}}, v_{2}\right.} \leq 2 C 2^{-\mu_{2}}} d \mu_{1}(s) d \mu_{2}(t) \\
& \times \frac{2^{k_{1}} 2^{-k_{1} \epsilon_{1}}}{\left(1+2^{\mu_{1}} \rho_{1}\left(x, z_{\tau_{1}}^{\mu_{1}, v_{1}}\right)\right)^{1+\epsilon_{1}}} \\
& \times \frac{2^{-\mu_{2} \epsilon_{2}} 2^{k_{2}} 2^{-k_{2} \epsilon_{2}}}{\left(1+2^{k_{2}} \rho_{2}\left(y, z_{\tau_{2}}^{\mu_{2}, v_{2}}\right)\right)^{1+\epsilon_{2}}}
\end{aligned}
$$




$$
\begin{aligned}
\leq & C \mu_{1}\left(Q_{\tau_{1}}^{\mu_{1}, v_{1}}\right)^{-1 / 2} \mu_{2}\left(Q_{\tau_{2}}^{\mu_{2}, v_{2}}\right)^{-1 / 2} \\
& \times \frac{2^{-\left(k_{1}-\mu_{1}\right) \epsilon_{1}}}{\left(1+2^{\mu_{1}} \rho_{1}\left(x, z_{\tau_{1}}^{\mu_{1}, v_{1}}\right)\right)^{1+\epsilon_{1}}} \\
& \times \frac{2^{-\left(\mu_{2}-k_{2}\right)\left(1+\epsilon_{2}\right)}}{\left(1+2^{k_{2}} \rho_{2}\left(y, z_{\tau_{2}}^{\mu_{2}, v_{2}}\right)\right)^{1+\epsilon_{2}}} .
\end{aligned}
$$

The estimate of the case $k_{1} \leq \mu_{1}, k_{2}>\mu_{2}$ is similar to the case $k_{1}>\mu_{1}, k_{2} \leq \mu_{2}$ by symmetry and we obtain

$$
\begin{aligned}
\left|D_{k_{1}} D_{k_{2}}\left(a_{R}\right)(x, y)\right| & \\
\leq & C \mu_{1}\left(Q_{\tau_{1}}^{\mu_{1}, v_{1}}\right)^{-1 / 2} \mu_{2}\left(Q_{\tau_{2}}^{\mu_{2}, v_{2}}\right)^{-1 / 2} \\
\quad & \times \frac{2^{-\left(\mu_{1}-k_{1}\right)\left(1+\epsilon_{1}\right)}}{\left(1+2^{k_{1}} \rho_{1}\left(y, z_{\tau_{1}}^{\mu_{1}, v_{1}}\right)\right)^{1+\epsilon_{1}}} \frac{2^{-\left(k_{2}-\mu_{2}\right) \epsilon_{2}}}{\left(1+2^{\mu_{2}} \rho_{2}\left(x, z_{\tau_{2}}^{\mu_{2}, v_{2}}\right)\right)^{1+\epsilon_{2}}} .
\end{aligned}
$$

For $k_{1} \leq \mu_{1}, k_{2} \leq \mu_{2}$, when $\rho_{1}\left(s, z_{\tau_{1}}^{u_{1}, v_{1}}\right) \leq(1 / 2 A)\left(2^{-k_{1}}+\right.$ $\left.\rho_{1}(s, x)\right)$ and $\rho_{2}\left(t, z_{\tau_{2}}^{u_{2}, v_{2}}\right) \leq(1 / 2 A)\left(2^{-k_{2}}+\rho_{2}(y, t)\right)$, then

$$
\begin{aligned}
&\left|D_{k_{1}} D_{k_{2}}\left(a_{R}\right)(x, y)\right| \\
&=\mid \int_{X_{1} \times X_{2}} {\left[D_{k_{1}}(x, s)-D_{k_{1}}\left(x, z_{\tau_{1}}^{u_{1}, v_{1}}\right)\right] } \\
& \times {\left[D_{k_{2}}(y, t)-D_{k_{2}}\left(y, z_{\tau_{2}}^{u_{2}, v_{2}}\right)\right] } \\
& \times a_{R}(s, t) d \mu_{1}(s) d \mu_{2}(t) \mid \\
& \leq C \mu_{1}\left(Q_{\tau_{1}}^{\mu_{1}, v_{1}}\right)^{-1 / 2} \mu_{2}\left(Q_{\tau_{2}}^{\mu_{2}, v_{2}}\right)^{-1 / 2}
\end{aligned}
$$

$$
\times \int_{\substack{\rho_{1}\left(s, z_{\tau_{1}, v_{1}}^{u_{1}}\right) \leq 2 C 2^{-\mu_{1}} \\ \rho_{2}\left(t, z_{\tau_{2}}^{u_{2}, v_{2}}\right) \leq 2 C 2^{-\mu_{2}}}}\left(\frac{\rho_{1}\left(s, z_{\tau_{1}}^{u_{1}, v_{1}}\right)}{2^{-k_{1}}+\rho_{1}(x, s)}\right)^{\epsilon_{1}}
$$$$
\times \frac{2^{-k_{1} \epsilon_{1}}}{\left(2^{-k_{1}}+\rho_{1}(x, s)\right)^{1+\epsilon_{1}}}\left(\frac{\rho_{2}\left(t, z_{\tau_{2}}^{u_{2}, v_{2}}\right)}{2^{-k_{2}}+\rho_{2}(t, y)}\right)^{\epsilon_{2}}
$$$$
\times \frac{2^{-k_{2} \epsilon_{2}}}{\left(2^{-k_{2}}+\rho_{2}(t, y)\right)^{1+\epsilon_{2}}} d \mu_{1}(s) d \mu_{2}(t)
$$$$
\leq C \mu_{1}\left(Q_{\tau_{1}}^{\mu_{1}, v_{1}}\right)^{-1 / 2} \mu_{2}\left(Q_{\tau_{2}}^{\mu_{2}, v_{2}}\right)^{-1 / 2}
$$$$
\times \int_{\substack{\rho_{1}\left(s, z_{\tau_{1}}^{u_{1}, v_{1}}\right) \leq 2 C 2^{-\mu_{1}} \\ \rho_{2}\left(t, z_{\tau_{2}, v_{2}}^{u_{2}}\right) \leq 2 C 2^{-\mu_{2}}}} \frac{2^{-\mu_{1} \epsilon_{1}}}{\left(2^{-k_{1}}+\rho_{1}(x, s)\right)^{\epsilon_{1}}}
$$$$
\times \frac{2^{k_{1}}}{\left(1+2^{k_{1}} \rho_{1}(x, s)\right)^{1+\epsilon_{1}}}
$$$$
\times \frac{2^{-\mu_{2} \epsilon_{2}}}{\left(2^{-k_{2}}+\rho_{2}(t, y)\right)^{\epsilon_{2}}}
$$$$
\times \frac{2^{k_{2}}}{\left(1+2^{k_{2}} \rho_{2}(t, y)\right)^{1+\epsilon_{2}}} d \mu_{1}(s) d \mu_{2}(t)
$$$$
\leq C \mu_{1}\left(Q_{\tau_{1}}^{\mu_{1}, v_{1}}\right)^{-1 / 2} \mu_{2}\left(Q_{\tau_{2}}^{\mu_{2}, v_{2}}\right)^{-1 / 2}
$$

$$
\begin{aligned}
& \times \frac{2^{-\left(\mu_{1}-k_{1}\right)\left(1+\epsilon_{1}\right)}}{\left(1+2^{k_{1}} \rho_{1}\left(x, z_{\tau_{1}}^{\mu_{1}, v_{1}}\right)\right)^{1+\epsilon_{1}}} \\
& \times \frac{2^{-\left(\mu_{2}-k_{2}\right)\left(1+\epsilon_{2}\right)}}{\left(1+2^{k_{2}} \rho_{2}\left(y, z_{\tau_{2}}^{\mu_{2}, v_{2}}\right)\right)^{1+\epsilon_{2}}} .
\end{aligned}
$$

We can similarly get the same result for another three cases; here we omit the details.

Thus, if $f=\sum_{\mu_{1}, \mu_{2} \in \mathbb{Z}} \sum_{\tau_{1} \in I_{\mu_{1}}} \sum_{v_{1}=1}^{N\left(\mu_{1}, \tau_{1}\right)} \sum_{\tau_{2} \in I_{\mu_{2}}} \sum_{v_{2}=1}^{N\left(\mu_{2}, \tau_{2}\right)} s_{R} a_{R}$, by Lemma 13 and above estimates, then

$$
\begin{aligned}
& \left\{\sum_{k_{1}, k_{2} \in \mathbb{Z}}\left(2^{k_{1} s_{1}} 2^{k_{2} s_{2}}\left\|D_{k_{1}} D_{k_{2}}(f)\right\|_{p}\right)^{q}\right\}^{1 / q} \\
& \quad=\left\{\sum_{k_{1}, k_{2} \in \mathbb{Z}}\left(2^{k_{1} s_{1}} 2^{k_{2} s_{2}}\left\|D_{k_{1}} D_{k_{2}}\left(\sum_{\mu_{1}, \mu_{2}} \sum_{\tau_{1} \in I_{\mu_{1}}} \sum_{v_{1}=1}^{N\left(\mu_{1}, \tau_{1}\right)} \sum_{\tau_{2} \in I_{\mu_{2}}}^{N\left(\mu_{2}, \tau_{2}\right)} \sum_{v_{2}=1} s_{R} a_{R}\right)\right\|_{p}\right)^{q}\right\}^{1 / q} \\
& \leq C\left\{\sum _ { k _ { 1 } , k _ { 2 } \in \mathbb { Z } } \left(\sum_{\mu_{1}=-\infty}^{k_{1}} \sum_{\mu_{2}=-\infty}^{k_{2}} 2^{k_{1} s_{1}} 2^{k_{2} s_{2}} 2^{\mu_{1} / 2} 2^{\mu_{2} / 2} 2^{\left(\mu_{1}-k_{1}\right) \epsilon_{1}} 2^{\left(\mu_{2}-k_{2}\right) \epsilon_{2}}\right.\right. \\
& \left.\left.\times M_{2}\left(\sum_{\tau_{2} \in I_{\mu_{2}}}^{N\left(\mu_{2}, \tau_{2}\right)} \sum_{v_{2}=1} M_{1}\left(\sum_{\tau_{1} \in I_{\mu_{1}}}^{N\left(\mu_{1}, \tau_{1}\right)}\left|\sum_{v_{1}=1}\right| s_{R} \mid \chi_{Q_{\tau_{1}}^{\mu_{1}, v_{1}}}\right) \chi_{Q_{\tau_{2}}^{\mu_{2}, v_{2}}}\right) \|_{p}\right)^{q}\right\}^{1 / q}
\end{aligned}
$$




$$
\begin{aligned}
& +C\left\{\sum _ { k _ { 1 } , k _ { 2 } \in \mathbb { Z } } \left(\sum_{\mu_{1}=-\infty}^{k_{1}} \sum_{\mu_{2}=k_{2}+1}^{\infty} 2^{k_{1} s_{1}} 2^{k_{2} s_{2}} 2^{\mu_{1} / 2} 2^{\mu_{2} / 2} 2^{\left(\mu_{1}-k_{1}\right) \epsilon_{1}} 2^{-\left(\mu_{2}-k_{2}\right)} 2^{\left(1+\epsilon_{2}\right)}\right.\right. \\
& \left.\left.\times\left\|2^{\left(\mu_{2}-k_{2}\right)} M_{2}\left(\sum_{\tau_{2} \in I_{\mu_{2}}} \sum_{v_{2}=1}^{N\left(\mu_{2}, \tau_{2}\right)} M_{1}\left(\sum_{\tau_{1} \in I_{\mu_{1}}} \sum_{v_{1}=1}^{N\left(\mu_{1}, \tau_{1}\right)}\left|s_{R}\right| \chi_{Q_{\tau_{1}}} \mu_{1, v_{1}}\right) \chi_{Q_{\tau_{2}}^{\mu_{2}, v_{2}}}\right)\right\|_{p}\right)^{q}\right\}^{1 / q} \\
& +C\left\{\sum _ { k _ { 1 } , k _ { 2 } \in \mathbb { Z } } \left(\sum_{\mu_{1}=k_{1}+1}^{\infty} \sum_{\mu_{2}=-\infty}^{k_{2}} 2^{k_{1} s_{1}} 2^{k_{2} s_{2}} 2^{\mu_{1} / 2} 2^{\mu_{2} / 2} 2^{-\left(\mu_{1}-k_{1}\right)\left(1+\epsilon_{1}\right)} 2^{\left(\mu_{2}-k_{2}\right) \epsilon_{2}}\right.\right. \\
& \left.\left.\times\left\|2^{\left(\mu_{1}-k_{1}\right)} M_{2}\left(\sum_{\tau_{2} \in I_{\mu_{2}}} \sum_{v_{2}=1}^{N\left(\mu_{2}, \tau_{2}\right)} M_{1}\left(\sum_{\tau_{1} \in I_{\mu_{1}}} \sum_{v_{1}=1}^{N\left(\mu_{1}, \tau_{1}\right)}\left|s_{R}\right| \chi_{Q_{\tau_{1}}^{\mu_{1}, \nu_{1}}}\right) \chi_{Q_{\tau_{2}}^{\mu_{2}, \nu_{2}}}\right)\right\|_{p}\right)^{q}\right\}^{1 / q} \\
& +C\left\{\sum _ { k _ { 1 } , k _ { 2 } \in \mathbb { Z } } \left(\sum_{\mu_{1}=k_{1}+1}^{\infty} \sum_{\mu_{2}=k_{2}+1}^{\infty} 2^{k_{1} s_{1}} 2^{k_{2} s_{2}} 2^{\mu_{1} / 2} 2^{\mu_{2} / 2} 2^{-\left(\mu_{1}-k_{1}\right)\left(1+\epsilon_{1}\right)} 2^{-\left(\mu_{2}-k_{2}\right)\left(1+\epsilon_{2}\right)}\right.\right. \\
& \left.\left.\times\left\|2^{\left(\mu_{1}-k_{1}\right)} 2^{\left(\mu_{2}-k_{2}\right)} M_{2}\left(\sum_{\tau_{2} \in I_{\mu_{2}}} \sum_{v_{2}=1}^{N\left(\mu_{2}, \tau_{2}\right)} M_{1}\left(\sum_{\tau_{1} \in I_{\mu_{1}}} \sum_{v_{1}=1}^{N\left(\mu_{1}, \tau_{1}\right)}\left|s_{R}\right| \chi_{Q_{\tau_{1}}, v_{1}}\right) \chi_{Q_{\tau_{2}}^{\mu_{2}, v_{2}}}\right)\right\|_{p}\right)^{q}\right\}^{1 / q} \\
& :=I_{1}+I_{2}+I_{3}+I_{4} \text {. }
\end{aligned}
$$

Let $A_{\mu}=\left(\sum_{\tau_{1} \in I_{\mu_{1}}} \sum_{v_{1}=1}^{N\left(\mu_{1}, \tau_{1}\right)} \sum_{\tau_{2} \in I_{\mu_{2}}} \sum_{v_{2}=1}^{N\left(\mu_{2}, \tau_{2}\right)}\left|s_{R}\right|^{p} \chi_{R}\right)^{1 / p}$. We now consider $I_{1}$. By Hölder's inequality and noting that $k_{i} \geq$ $\mu_{i}, \epsilon_{i}>s_{i}$ for $i=1,2$, we have

$$
\begin{aligned}
I_{1} & \leq C\left\{\sum_{k_{1}, k_{2} \in \mathbb{Z}}\left(\sum_{\mu_{1}=-\infty}^{k_{1}} \sum_{\mu_{2}=-\infty}^{k_{2}} 2^{k_{1} s_{1}} 2^{k_{2} s_{2}} 2^{\mu_{1} / 2} 2^{\mu_{2} / 2} 2^{\left(\mu_{1}-k_{1}\right) \epsilon_{1}} 2^{\left(\mu_{2}-k_{2}\right) \epsilon_{2}} A_{\mu} 2^{-\mu_{1} / p} 2^{-\mu_{2} / p}\right)^{q}\right\}^{1 / q} \\
& \leq C\left\{\sum_{k_{1}, k_{2} \in \mathbb{Z}} \sum_{\mu_{1}=-\infty}^{k_{1}} \sum_{\mu_{2}=-\infty}^{k_{2}} 2^{-\left(k_{1}-\mu_{1}\right)\left(\epsilon_{1}-s_{1}\right)} 2^{-\left(k_{2}-\mu_{2}\right)\left(\epsilon_{2}-s_{2}\right)}\left(2^{\mu_{1}\left(1 / 2+s_{1}-1 / p\right)} 2^{\mu_{2}\left(1 / 2+s_{2}-1 / p\right)} A_{\mu}\right)^{q}\right\}^{1 / q} \\
& \leq C\left\{\sum_{\mu_{1}, \mu_{2} \in \mathbb{Z}}\left(2^{\mu_{1}\left(1 / 2+s_{1}-1 / p\right)} 2^{\mu_{2}\left(1 / 2+s_{2}-1 / p\right)} A_{\mu}\right)^{q}\right\}^{1 / q} \leq C\|S\|_{i_{p}^{s, q} .}
\end{aligned}
$$


Similar to the proof of $I_{1}$, we get desired results for $I_{2}, I_{3}$, and $I_{4}$.

To show the second inequality of (25), let $a(x, y)=$ $\left\{a_{\mu_{1}, \mu_{2}}(x, y)\right\}_{\mu_{1}, \mu_{2} \in \mathbb{Z}}$ with

$$
\begin{aligned}
& a_{\mu_{1}, \mu_{2}}(x, y) \\
& =M_{2}\left(\sum_{\tau_{2} \in I_{\mu_{2}}} \sum_{v_{2}=1}^{N\left(\mu_{2}, \tau_{2}\right)} \mu_{2}\left(Q_{\tau_{2}}^{\mu_{2}, v_{2}}\right)^{-1 / 2-s_{2}}\right. \\
& \quad \times M_{1}\left(\sum_{\tau_{1} \in I_{\mu_{1}}} \sum_{v_{1}=1}^{N\left(\mu_{1}, \tau_{1}\right)} \mu_{1}\left(Q_{\tau_{1}}^{\mu_{1}, v_{1}}\right)^{-1 / 2-s_{1}}\left|s_{R}\right| \chi_{Q_{\tau_{1}}^{\mu_{1}, v_{1}}}\right) \\
& \left.\quad \times(x) \chi_{Q_{\tau_{2}}^{\mu_{2}, v_{2}}}\right)(y) .
\end{aligned}
$$

Applying Lemma 13 and Hölder's inequality, we get

$$
\begin{aligned}
& \left\{\sum_{k_{1}, k_{2} \in \mathbb{Z}}\left(2^{k_{1} s_{1}} 2^{k_{2} s_{2}}\left|D_{k_{1}} D_{k_{2}}(f)\right|\right)^{q}\right\}^{1 / q} \\
& \leq C\left\{\sum _ { k _ { 1 } , k _ { 2 } \in \mathbb { Z } } \left(\sum_{\mu_{1}=-\infty}^{k_{1}} \sum_{\mu_{2}=-\infty}^{k_{2}} 2^{-\left(k_{1}-\mu_{1}\right)\left(\epsilon_{1}-s_{1}\right)} 2^{-\left(k_{2}-\mu_{2}\right)\left(\epsilon_{2}-s_{2}\right)}\right.\right. \\
& \left.\left.\times a_{\mu_{1}, \mu_{2}}(x, y)\right)^{q}\right\}^{1 / q} \\
& +C\left\{\sum _ { k _ { 1 } , k _ { 2 } \in \mathbb { Z } } \left(\sum_{\mu_{1}=-\infty}^{k_{1}} \sum_{\mu_{2}=k_{2}+1}^{\infty} 2^{-\left(k_{1}-\mu_{1}\right)\left(\epsilon_{1}-s_{1}\right)} 2^{\left(k_{2}-\mu_{2}\right)\left(\epsilon_{2}+s_{2}\right)}\right.\right. \\
& \left.\left.\times a_{\mu_{1}, \mu_{2}}(x, y)\right)^{q}\right\}^{1 / q} \\
& +C\left\{\sum _ { k _ { 1 } , k _ { 2 } \in \mathbb { Z } } \left(\sum_{\mu_{1}=k_{1}+1}^{\infty} \sum_{\mu_{2}=-\infty}^{k_{2}} 2^{\left(k_{1}-\mu_{1}\right)\left(\epsilon_{1}+s_{1}\right)} 2^{-\left(k_{2}-\mu_{2}\right)\left(\epsilon_{2}-s_{2}\right)}\right.\right. \\
& \left.\left.\times a_{\mu_{1}, \mu_{2}}(x, y)\right)^{q}\right\}^{1 / q} \\
& +C\left\{\sum _ { k _ { 1 } , k _ { 2 } \in \mathbb { Z } } \left(\sum_{\mu_{1}=k_{1}+1}^{\infty} \sum_{\mu_{2}=k_{2}+1}^{\infty} 2^{\left(k_{1}-\mu_{1}\right)\left(\epsilon_{1}+s_{1}\right)} 2^{\left(k_{2}-\mu_{2}\right)\left(\epsilon_{2}+s_{2}\right)}\right.\right. \\
& \left.\left.\times a_{\mu_{1}, \mu_{2}}(x, y)\right)^{q}\right\}^{1 / q} \\
& \leq C\left\{\sum_{\mu_{1}, \mu_{2} \in \mathbb{Z}} \sum_{k_{1}=\mu_{1}}^{\infty} \sum_{k_{2}=\mu_{2}}^{\infty} 2^{-\left(k_{1}-\mu_{1}\right)\left(\epsilon_{1}-s_{1}\right)} 2^{-\left(k_{2}-\mu_{2}\right)\left(\epsilon_{2}-s_{2}\right)}\right.
\end{aligned}
$$

$$
\left.\times a_{\mu_{1}, \mu_{2}}(x, y)^{q}\right\}^{1 / q}
$$$$
+C\left\{\sum_{\mu_{1}, \mu_{2} \in \mathbb{Z}} \sum_{k_{1}=\mu_{1}}^{\infty} \sum_{k_{2}=-\infty}^{\mu_{2}} 2^{-\left(k_{1}-\mu_{1}\right)\left(\epsilon_{1}-s_{1}\right)} 2^{\left(k_{2}-\mu_{2}\right)\left(\epsilon_{2}+s_{2}\right)}\right.
$$$$
\left.\times a_{\mu_{1}, \mu_{2}}(x, y)^{q}\right\}^{1 / q}
$$$$
+C\left\{\sum_{\mu_{1}, \mu_{2} \in \mathbb{Z}} \sum_{k_{1}=-\infty}^{\mu_{1}} \sum_{k_{2}=\mu_{2}}^{\infty} 2^{\left(k_{1}-\mu_{1}\right)\left(\epsilon_{1}+s_{1}\right)} 2^{-\left(k_{2}-\mu_{2}\right)\left(\epsilon_{2}-s_{2}\right)}\right.
$$$$
\left.\times a_{\mu_{1}, \mu_{2}}(x, y)^{q}\right\}^{1 / q}
$$$$
+C\left\{\sum_{\mu_{1}, \mu_{2} \in \mathbb{Z}} \sum_{k_{1}=-\infty}^{\mu_{1}} \sum_{k_{2}=-\infty}^{\mu_{2}} 2^{\left(k_{1}-\mu_{1}\right)\left(\epsilon_{1}+s_{1}\right)} 2^{\left(k_{2}-\mu_{2}\right)\left(\epsilon_{2}+s_{2}\right)}\right.
$$$$
\left.\times a_{\mu_{1}, \mu_{2}}(x, y)^{q}\right\}^{1 / q}
$$$$
\leq C\left\{\sum_{\mu_{1}, \mu_{2} \in \mathbb{Z}} a_{\mu_{1}, \mu_{2}}(x, y)^{q}\right\}^{1 / q} .
$$

Hence, by Fefferman-Stein vector-valued maximal inequality, we obtain

$$
\begin{aligned}
& \|f\|_{\dot{F}_{p}^{s, q}} \\
& =\left\|\left\{\sum_{k_{1}, k_{2} \in \mathbb{Z}}\left(2^{k_{1} s_{1}} 2^{k_{2} s_{2}}\left|D_{k_{1}} D_{k_{2}}(f)\right|\right)^{q}\right\}^{1 / q}\right\|_{p} \\
& \leq C \|\left\{\sum _ { \mu _ { 1 } , \mu _ { 2 } \in \mathbb { Z } } \left(\sum_{\tau_{2} \in I_{\mu_{1}}} \sum_{v_{1}=1}^{N\left(\mu_{1}, \tau_{1}\right)} \sum_{\tau_{2} \in I_{\mu_{2}}} \sum_{v_{2}=1}^{N\left(\mu_{2}, \tau_{2}\right)} \mu_{1}\left(Q_{\tau_{1}}^{\mu_{1}, v_{1}}\right)^{-1 / 2-s_{1}}\right.\right. \\
& \left.\left.\quad \times \mu_{2}\left(Q_{\tau_{2}}^{\mu_{2}, v_{2}}\right)^{-1 / 2-s_{2}}\left|s_{R}\right| \chi_{R}\right)^{q}\right\}^{1 / q} \|_{p} \\
& \leq C\|S\|_{f_{p}^{s, q},}
\end{aligned}
$$

which completes the proof of Theorem 12 .

\section{Smooth Molecular Decomposition}

In this section, we show smooth molecular decomposition of Triebel-Lizorkin and Besov spaces. We first give definitions of the smooth molecules as follows. 
Definition 14. Suppose that collections of open subset $\left\{Q_{\tau_{1}}^{k_{1}, v_{1}} \subset X_{1}: k_{1} \in \mathbb{Z}, \tau_{1} \in I_{k_{1}}, v_{1}=1, \ldots, N\left(k_{1}, \tau_{1}\right)\right\}$ and $\left\{Q_{\tau_{2}}^{k_{2}, v_{2}} \subset X_{2}: k_{2} \in \mathbb{Z}, \tau_{2} \in I_{k_{2}}, v_{2}=1, \ldots, N\left(k_{2}, \tau_{2}\right)\right\}$ satisfy the conditions in Lemma 5. Let $\beta=\left(\beta_{1}, \beta_{2}\right), \gamma=\left(\gamma_{1}, \gamma_{2}\right)$ and $R=Q_{\tau_{1}}^{k_{1}, v_{1}} \times Q_{\tau_{2}}^{k_{2}, v_{2}}$, and $z_{\tau_{1}}^{k_{1}, v_{1}}, z_{\tau_{1}}^{k_{1}, v_{1}}$ are the centers of $Q_{\tau_{1}}^{k_{1}, v_{1}}, Q_{\tau_{2}}^{k_{2}, v_{2}}$, respectively. A function $m_{R}$ defined on $X_{1} \times X_{2}$ is said to be a $(\beta, \gamma)$-smooth molecule for $R$ if

(i)

$$
\int_{X_{2}} m_{R}\left(x_{1}, x_{2}\right) d \mu_{2}\left(x_{2}\right)=\int_{X_{1}} m_{R}\left(x_{1}, x_{2}\right) d \mu_{1}\left(x_{1}\right)=0 ;
$$

(ii)

$$
\begin{aligned}
& \left|m_{R}(x, y)\right| \leq C \mu_{1}\left(Q_{\tau_{1}}^{k_{1}, v_{1}}\right)^{-1 / 2} \mu_{2}\left(Q_{\tau_{2}}^{k_{2}, v_{2}}\right)^{-1 / 2} \\
& \times\left(1+2^{-k_{1}} \rho_{1}\left(x, z_{\tau_{1}}^{k_{1}, v_{1}}\right)\right)^{-\left(1+\gamma_{1}\right)} \\
& \times\left(1+2^{-k_{2}} \rho_{2}\left(y, z_{\tau_{2}}^{k_{2}, v_{2}}\right)\right)^{-\left(1+\gamma_{2}\right)}, \\
& \left|m_{R}(x, y)-m_{R}\left(x^{\prime}, y\right)\right| \\
& \leq C \mu_{1}\left(Q_{\tau_{1}}^{k_{1}, v_{1}}\right)^{-1 / 2-\beta_{1}} \mu_{2}\left(Q_{\tau_{2}}^{k_{2}, v_{2}}\right)^{-1 / 2} \\
& \times \rho_{1}\left(x, x^{\prime}\right)^{\beta_{1}}\left(1+2^{-k_{2}} \rho_{2}\left(y, z_{\tau_{2}}^{k_{2}, v_{2}}\right)\right)^{\left(-1+\gamma_{2}\right)} \\
& \times\left\{\left(1+2^{-k_{1}} \rho_{1}\left(x, z_{\tau_{1}}^{k_{1}, v_{1}}\right)\right)^{-\left(1+\gamma_{1}\right)}\right. \\
& \left.+\left(1+2^{-k_{1}} \rho_{1}\left(x^{\prime}, z_{\tau_{1}}^{k_{1}, v_{1}}\right)\right)^{-\left(1+\gamma_{1}\right)}\right\}, \\
& \left|m_{R}(x, y)-m_{R}\left(x, y^{\prime}\right)\right| \\
& \leq C \mu_{1}\left(Q_{\tau_{1}}^{k_{1}, v_{1}}\right)^{-1 / 2} \mu_{2}\left(Q_{\tau_{2}}^{k_{2}, v_{2}}\right)^{-1 / 2-\beta_{2}} \\
& \times \rho_{2}\left(y, y^{\prime}\right)^{\beta_{2}}\left(1+2^{-k_{1}} \rho_{1}\left(x, z_{\tau_{1}}^{k_{1}, v_{1}}\right)\right)^{-\left(1+\gamma_{1}\right)} \\
& \times\left\{\left(1+2^{-k_{2}} \rho_{2}\left(y, z_{\tau_{2}}^{k_{2}, v_{2}}\right)\right)^{-\left(1+\gamma_{2}\right)}\right. \\
& \left.+\left(1+2^{-k_{2}} \rho_{2}\left(y^{\prime}, z_{\tau_{2}}^{k_{2}, v_{2}}\right)\right)^{-\left(1+\gamma_{2}\right)}\right\}, \\
& \left|m_{R}(x, y)-m_{R}\left(x^{\prime}, y\right)-m_{R}\left(x, y^{\prime}\right)-m_{R}\left(x^{\prime}, y^{\prime}\right)\right| \\
& \leq C \mu_{1}\left(Q_{\tau_{1}}^{k_{1}, v_{1}}\right)^{-1 / 2} \mu_{2}\left(Q_{\tau_{2}}^{k_{2}, v_{2}}\right)^{-1 / 2} \\
& \times \rho_{1}\left(x, x^{\prime}\right)^{\beta_{1}} \rho_{2}\left(y, y^{\prime}\right)^{\beta_{2}} \\
& \times\left\{\left(1+2^{-k_{1}} \rho_{1}\left(x, z_{\tau_{1}}^{k_{1}, v_{1}}\right)\right)^{-\left(1+\gamma_{1}\right)}\right. \\
& \left.+\left(1+2^{-k_{1}} \rho_{1}\left(x^{\prime}, z_{\tau_{1}}^{k_{1}, v_{1}}\right)\right)^{-\left(1+\gamma_{1}\right)}\right\}
\end{aligned}
$$

Now we can state the smooth molecular decomposition for Triebel-Lizorkin and Besov spaces.

Theorem 15. Let $R$ be as in Definition 11. Suppose that for $i=$ $1,2, s=\left(s_{1}, s_{2}\right), \epsilon=\left(\epsilon_{1}, \epsilon_{2}\right), \beta=\left(\beta_{1}, \beta_{2}\right), \gamma=\left(\gamma_{1}, \gamma_{2}\right),-\epsilon_{i}<$ $s_{i}<\epsilon_{i}, 1<p, q<\infty, m_{R}$ is a $(\beta, \gamma)$-smooth molecule with $\max \left(0, s_{i}\right)<\beta_{i}<\epsilon_{i}$ and $\max \left(0,-s_{i}\right)<\gamma_{i}<\epsilon_{i}$ for $i=1$, 2. If

$$
f=\sum_{k_{1}, k_{2} \in \mathbb{Z}} \sum_{\tau_{1} \in I_{k_{1}}} \sum_{v_{1}=1}^{N\left(k_{1}, \tau_{1}\right)} \sum_{\tau_{2} \in I_{k_{2}}} \sum_{v_{2}=1}^{N\left(k_{2}, \tau_{2}\right)} s_{R} m_{R},
$$

then $\|f\|_{\dot{B}_{p}^{s, q}} \leq C\|S\|_{\dot{b}_{p}^{s, q}}$ and $\|f\|_{\dot{F}_{p}^{s, q}} \leq C\|S\|_{\dot{f}_{p}^{s, q}}$.

Proof. Suppose that $R=Q_{\tau_{1}}^{\mu_{1}, v_{1}} \times Q_{\tau_{2}}^{\mu_{2}, v_{2}}$. From the proof of Theorem 12, we only need to claim the following estimates. If $k_{1} \leq \mu_{1}, k_{1} \leq \mu_{2}$, then

$$
\begin{aligned}
& \left|D_{k_{1}} D_{k_{2}}\left(m_{R}\right)(x, y)\right| \\
& \leq C \\
& \quad C \mu_{1}\left(Q_{\tau_{1}}^{\mu_{1}, v_{1}}\right)^{-1 / 2} \mu_{2}\left(Q_{\tau_{2}}^{\mu_{2}, v_{2}}\right)^{-1 / 2} \\
& \quad \times \frac{2^{-\left(\mu_{1}-k_{1}\right)\left(1+\gamma_{1}\right)}}{\left(1+2^{k_{1}} \rho_{1}\left(x, z_{\tau_{1}}^{\mu_{1}, v_{1}}\right)\right)^{1+\gamma_{1}}} \frac{2^{-\left(\mu_{2}-k_{2}\right)\left(1+\gamma_{2}\right)}}{\left(1+2^{k_{2}} \rho_{2}\left(y, z_{\tau_{2}}^{\mu_{2}, \nu_{2}}\right)\right)^{1+\gamma_{2}}} .
\end{aligned}
$$

If $k_{1}>\mu_{1}, k_{1} \leq \mu_{2}$, then

$$
\begin{aligned}
\left|D_{k_{1}} D_{k_{2}}\left(m_{R}\right)(x, y)\right| \\
\leq \\
\leq C \mu_{1}\left(Q_{\tau_{1}}^{\mu_{1}, v_{1}}\right)^{-1 / 2} \mu_{2}\left(Q_{\tau_{2}}^{\mu_{2}, v_{2}}\right)^{-1 / 2} \\
\quad \times \frac{2^{-\left(k_{1}-\mu_{1}\right) \beta_{1}}}{\left(1+2^{\mu_{1}} \rho_{1}\left(x, z_{\tau_{1}}^{\mu_{1}, v_{1}}\right)\right)^{1+\gamma_{1}}} \frac{2^{-\left(\mu_{2}-k_{2}\right)\left(1+\gamma_{2}\right)}}{\left(1+2^{k_{2}} \rho_{2}\left(y, z_{\tau_{2}}^{\mu_{2}, v_{2}}\right)\right)^{1+\gamma_{2}}} .
\end{aligned}
$$

If $k_{1} \leq \mu_{1}, k_{1}>\mu_{2}$, then

$$
\begin{aligned}
\left|D_{k_{1}} D_{k_{2}}\left(m_{R}\right)(x, y)\right| \\
\leq \\
\leq C \mu_{1}\left(Q_{\tau_{1}}^{\mu_{1}, v_{1}}\right)^{-1 / 2} \mu_{2}\left(Q_{\tau_{2}}^{\mu_{2}, v_{2}}\right)^{-1 / 2} \\
\quad \times \frac{2^{-\left(\mu_{1}-k_{1}\right)\left(1+\gamma_{1}\right)}}{\left(1+2^{k_{1}} \rho_{1}\left(x, z_{\tau_{1}}^{\mu_{1}, v_{1}}\right)\right)^{1+\gamma_{1}}} \frac{2^{-\left(k_{2}-\mu_{2}\right) \beta_{2}}}{\left(1+2^{\mu_{2}} \rho_{2}\left(y, z_{\tau_{2}}^{\mu_{2}, v_{2}}\right)\right)^{1+\gamma_{2}}} .
\end{aligned}
$$


If $k_{1}>\mu_{1}, k_{1}>\mu_{2}$, then

$$
\begin{aligned}
&\left|D_{k_{1}} D_{k_{2}}\left(m_{R}\right)(x, y)\right| \\
& \leq C \mu_{1}\left(Q_{\tau_{1}}^{\mu_{1}, v_{1}}\right)^{-1 / 2} \mu_{2}\left(Q_{\tau_{2}}^{\mu_{2}, v_{2}}\right)^{-1 / 2} \\
& \quad \times \frac{2^{-\left(k_{1}-\mu_{1}\right) \beta_{1}}}{\left(1+2^{\mu_{1}} \rho_{1}\left(x, z_{\tau_{1}}^{\mu_{1}, v_{1}}\right)\right)^{1+\gamma_{1}}} \frac{2^{-\left(k_{2}-\mu_{2}\right) \beta_{2}}}{\left(1+2^{\mu_{2}} \rho_{2}\left(y, z_{\tau_{2}}^{\mu_{2}, v_{2}}\right)\right)^{1+\gamma_{2}}} .
\end{aligned}
$$

We now prove the above estimates. Let $W_{1}=\{x$ : $\left.\rho_{1}\left(x, z_{\tau_{1}}^{\mu_{1}, v_{1}}\right) \leq 4 C_{1} 2^{-k_{1}}\right\}, W_{2}=\left\{y: \rho_{2}\left(y, z_{\tau_{2}}^{\mu_{2}, v_{2}}\right) \leq 4 C_{2} 2^{-k_{2}}\right\}$, $W_{3}=\left\{x: \rho_{1}(x, s) \leq 4 C_{1} 2^{-k_{1}}\right\}$, and $W_{4}=\left\{y: \rho_{2}(y, t) \leq\right.$ $\left.4 C_{2} 2^{-k_{2}}\right\}$, where $2 C_{i}$ is the smallest constant which satisfies supp $D_{k_{i}}(x, y) \subset\left\{(x, y) \in X_{i} \times X_{i}: \rho_{i}(x, y) \leq 2 C_{i} 2^{-k_{i}}\right\}$ for $i=1,2$, and $W_{1}^{C}, W_{2}^{C}, W_{3}^{C}$, and $W_{4}^{C}$ are their complements, respectively. We first give the proof of (48). Note that $k_{1} \leq \mu_{1}$, $k_{2} \leq \mu_{2}$, and then

$$
\begin{aligned}
& \left|D_{k_{1}} D_{k_{2}}\left(m_{R}\right)(x, y)\right| \\
& \leq \int_{X_{1} \times X_{2}}\left|D_{k_{1}}(x, s)-D_{k_{1}}\left(x, z_{\tau_{1}}^{\mu_{1}, v_{1}}\right)\right| \\
& \times\left|D_{k_{2}}(y, t)-D_{k_{2}}\left(y, z_{\tau_{2}}^{\mu_{2}, v_{2}}\right)\right| \\
& \times\left|m_{R}(s, t)\right| d \mu_{1}(s) \mu_{2}(t) \\
& \leq\left(\chi_{W_{1}} \chi_{W_{2}} \int_{W_{3} \times W_{4}}+\chi_{W_{1}^{c}} \chi_{W_{2}} \int_{W_{3} \times W_{4}}\right. \\
& +\chi_{W_{1}} \chi_{W_{2}^{C}} \int_{W_{3} \times W_{4}}+\chi_{W_{1}} \chi_{W_{2}} \int_{W_{3}^{C} \times W_{4}} \\
& +\chi_{W_{1}} \chi_{W_{2}} \int_{W_{3} \times W_{4}^{C}}+\chi_{W_{1}^{C}} \chi_{W_{2}^{C}} \int_{W_{3} \times W_{4}} \\
& +\chi_{W_{1}^{C}} \chi_{W_{2}} \int_{W_{3}^{C} \times W_{4}}+\chi_{W_{1}^{C}} \chi_{W_{2}} \int_{W_{3} \times W_{4}^{C}} \\
& +\chi_{W_{1}} \chi_{W_{2}^{C}} \int_{W_{3}^{C} \times W_{4}}+\chi_{W_{1}} \chi_{W_{2}^{C}} \int_{W_{3} \times W_{4}^{C}} \\
& +\chi_{W_{1}} \chi_{W_{2}} \int_{W_{3}^{C} \times W_{4}^{C}}+\chi_{W_{1}^{C}} \chi_{W_{2}^{C}} \int_{W_{3}^{C} \times W_{4}} \\
& +\chi_{W_{1}^{C}} \chi_{W_{2}^{C}} \int_{W_{3} \times W_{4}^{C}}+\chi_{W_{1}^{C}} \chi_{W_{2}} \int_{W_{3}^{C} \times W_{4}^{C}} \\
& \left.+\chi_{W_{1}} \chi_{W_{2}^{C}} \int_{W_{3}^{C} \times W_{4}^{C}}+\chi_{W_{1}^{C}} \chi_{W_{2}^{C}} \int_{W_{3}^{C} \times W_{4}^{C}}\right) \\
& \times\left|D_{k_{1}}(x, s)-D_{k_{1}}\left(x, z_{\tau_{1}}^{\mu_{1}, v_{1}}\right)\right| \\
& \times\left|D_{k_{2}}(y, t)-D_{k_{2}}\left(y, z_{\tau_{2}}^{\mu_{2}, v_{2}}\right)\right| \\
& \times\left|m_{R}(s, t)\right| d \mu_{1}(s) \mu_{2}(t) \\
& :=(48)_{1}+(48)_{2}+\cdots+(48)_{16} \text {. }
\end{aligned}
$$

By the support of $D_{k_{i}}$ and the definition of $W_{j}$ for $i=1,2$ and $j=1,2,3,4$, we can easily get $(48)_{7}=(48)_{10}=(48)_{12}=$ $(48)_{13}=(48)_{14}=(48)_{15}=(48)_{16}=0$. Here we only estimate the first five terms, and we can similarly estimate other terms. Now, we first prove $(48)_{1}$ :

$$
\begin{aligned}
& \left|(48)_{1}\right| \leq C 2^{k_{1}\left(1+\epsilon_{1}\right)} 2^{k_{2}\left(1+\epsilon_{2}\right)} \\
& \times \mu_{1}\left(Q_{\tau_{1}}^{\mu_{1}, v_{1}}\right)^{-1 / 2} \mu_{2}\left(Q_{\tau_{2}}^{\mu_{2}, v_{2}}\right)^{-1 / 2} \chi_{W_{1}} \chi_{W_{2}} \\
& \times \int_{W_{3} \times W_{4}} \frac{\rho_{1}\left(s, z_{\tau_{1}}^{\mu_{1}, v_{1}}\right)^{\epsilon_{1}}}{\left(1+2^{\mu_{1}} \rho_{1}\left(s, z_{\tau_{1}}^{\mu_{1}, v_{1}}\right)\right)^{\left(1+\gamma_{1}\right)}} \\
& \times \frac{\rho_{2}\left(t, z_{\tau_{2}}^{\mu_{2}, v_{2}}\right)^{\epsilon_{2}}}{\left(1+2^{\mu_{2}} \rho_{2}\left(t, z_{\tau_{2}}^{\mu_{2}, v_{2}}\right)\right)^{\left(1+\gamma_{2}\right)}} d \mu_{1}(s) d \mu_{2}(t) \\
& \leq C \mu_{1}\left(Q_{\tau_{1}}^{\mu_{1}, v_{1}}\right)^{-1 / 2} \mu_{2}\left(Q_{\tau_{2}}^{\mu_{2}, v_{2}}\right)^{-1 / 2} \\
& \times 2^{k_{1}\left(1+\epsilon_{1}\right)} 2^{k_{2}\left(1+\epsilon_{2}\right)} 2^{-\mu_{1}\left(1+\gamma_{1}\right)} 2^{\mu_{2}\left(1+\gamma_{2}\right)} \chi_{W_{1}} \chi_{W_{2}} \\
& \times \int_{\rho_{1}\left(s, z_{\tau_{1}}^{\mu_{1}, v_{1}}\right) \leq 8 C_{1} 2^{-k_{1}}} \rho_{1}\left(s, z_{\tau_{1}}^{\mu_{1}, v_{1}}\right)^{\epsilon_{1}-\gamma_{1}-1} d \mu_{1}(s) \\
& \times \int_{\rho_{2}\left(t, z_{\tau_{2}}^{\mu_{2}, v_{2}}\right) \leq 8 C_{2} 2^{-k_{2}}} \rho_{1}\left(t, z_{\tau_{2}}^{\mu_{2}, v_{2}}\right)^{\epsilon_{2}-\gamma_{2}-1} d \mu_{2}(t) \\
& \leq C \mu_{1}\left(Q_{\tau_{1}}^{\mu_{1}, v_{1}}\right)^{-1 / 2} \mu_{2}\left(Q_{\tau_{2}}^{\mu_{2}, v_{2}}\right)^{-1 / 2} \\
& \times 2^{-\left(\mu_{1}-k_{1}\right)\left(1+\gamma_{1}\right)} 2^{-\left(\mu_{2}-k_{2}\right)\left(1+\gamma_{2}\right)} \chi_{W_{1}} \chi_{W_{2}} \\
& \leq C \mu_{1}\left(Q_{\tau_{1}}^{\mu_{1}, v_{1}}\right)^{-1 / 2} \mu_{2}\left(Q_{\tau_{2}}^{\mu_{2}, v_{2}}\right)^{-1 / 2} \\
& \times \frac{2^{-\left(\mu_{1}-k_{1}\right)\left(1+\gamma_{1}\right)}}{\left(1+2^{k_{1}} \rho_{1}\left(x, z_{\tau_{1}}^{\mu_{1}, v_{1}}\right)\right)^{1+\gamma_{1}}} \\
& \times \frac{2^{-\left(\mu_{2}-k_{2}\right)\left(1+\gamma_{2}\right)}}{\left(1+2^{k_{2}} \rho_{2}\left(y, z_{\tau_{2}}^{\mu_{2}, v_{2}}\right)\right)^{1+\gamma_{2}}},
\end{aligned}
$$

which is the desired estimate.

We now estimate $(48)_{2}$ by

$\left|(48)_{2}\right|$

$$
\begin{gathered}
\leq C 2^{k_{1}} 2^{k_{2}\left(1+\epsilon_{2}\right)} \mu_{1}\left(Q_{\tau_{1}}^{\mu_{1}, v_{1}}\right)^{-1 / 2} \mu_{2}\left(Q_{\tau_{2}}^{\mu_{2}, v_{2}}\right)^{-1 / 2} \chi_{W_{1}^{C}} \chi_{W_{2}} \\
\times \int_{\substack{\rho_{1}(x, s) \\
\leq 2 C_{1} 2^{-k_{1}}}}\left(1 \times\left(\left(1+2^{\mu_{1}}\right.\right.\right. \\
\rho_{1}\left(s, z_{\tau_{1}}^{\mu_{1}, v_{1}}\right) \\
\geq \rho_{1}\left(x, z_{\tau_{1}}^{\mu_{1}, v_{1}}\right) / 2
\end{gathered}
$$$$
\left.\left.\left.\times \rho_{1}\left(s, z_{\tau_{1}}^{\mu_{1}, v_{1}}\right)\right)^{\left(1+\gamma_{1}\right)}\right)^{-1}\right) d \mu_{1}(s)
$$ 
Journal of Function Spaces

13

$$
\begin{aligned}
& \times \int_{W_{4}} \frac{\rho_{2}\left(t, z_{\tau_{2}}^{\mu_{2}, v_{2}}\right)^{\epsilon_{2}}}{\left(1+2^{\mu_{2}} \rho_{2}\left(t, z_{\tau_{2}}^{\mu_{2}, v_{2}}\right)\right)^{\left(1+\gamma_{2}\right)}} d \mu_{2}(t) \\
\leq & C 2^{k_{1}} 2^{k_{2}\left(1+\epsilon_{2}\right)} \mu_{1}\left(Q_{\tau_{1}}^{\mu_{1}, v_{1}}\right)^{-1 / 2} \\
& \times \mu_{2}\left(Q_{\tau_{2}}^{\mu_{2}, v_{2}}\right)^{-1 / 2} \chi_{W_{1}^{c}} \chi_{W_{2}} \\
& \times \frac{2^{-k_{1}}}{2^{\mu_{1}\left(1+\gamma_{1}\right)} \rho_{1}\left(x, z_{\tau_{1}}^{\mu_{1}, v_{1}}\right)^{\left(1+\gamma_{1}\right)}} 2^{-\mu_{2}\left(1+\gamma_{2}\right)} \\
& \times \int_{W_{4}} \rho_{2}\left(t, z_{\tau_{2}}^{\mu_{2}, v_{2}}\right)^{\epsilon_{2}-1-\gamma} d \mu_{2}(t) \\
\leq & C \mu_{1}\left(Q_{\tau_{1}}^{\mu_{1}, v_{1}}\right)^{-1 / 2} \mu_{2}\left(Q_{\tau_{2}}^{\mu_{2}, v_{2}}\right)^{-1 / 2} \\
& \times \frac{2^{-\left(\mu_{1}-k_{1}\right)\left(1+\gamma_{1}\right)}}{\left(1+2^{k_{1}} \rho_{1}\left(x, z_{\tau_{1}}^{\mu_{1}, v_{1}}\right)\right)^{1+\gamma_{1}}} \\
& \times \frac{2^{-\left(\mu_{2}-k_{2}\right)\left(1+\gamma_{2}\right)}}{\left(1+2^{k_{2}} \rho_{2}\left(y, z_{\tau_{2}}^{\mu_{2}, v_{2}}\right)\right)^{1+\gamma_{2}}},
\end{aligned}
$$

which verifies $(48)_{2}$.

The verification of $(48)_{3}$ is similar to that of $(48)_{2}$ by symmetry.

Similarly we have

$$
\begin{aligned}
& \left|(48)_{4}\right| \leq C 2^{k_{1}} 2^{k_{2}\left(1+\epsilon_{2}\right)} \mu_{1}\left(Q_{\tau_{1}}^{\mu_{1}, v_{1}}\right)^{-1 / 2} \mu_{2}\left(Q_{\tau_{2}}^{\mu_{2}, v_{2}}\right)^{-1 / 2} \chi_{W_{1}} \chi_{W_{2}} \\
& \times \int_{\rho_{1}\left(s, z_{\tau_{1}}^{\mu_{1}, v_{1}}\right) \geq 2 C_{1} 2^{-k_{1}}} \frac{1}{\left(2^{\mu_{1}} \rho_{1}\left(s, z_{\tau_{1}}^{\mu_{1}, v_{1}}\right)\right)^{\left(1+\gamma_{1}\right)}} d \mu_{1}(s) \\
& \times \int_{W_{4}} \frac{\rho_{2}\left(t, z_{\tau_{2}}^{\mu_{2}, v_{2}}\right)^{\epsilon_{2}}}{\left(1+2^{\mu_{2}} \rho_{2}\left(t, z_{\tau_{2}}^{\mu_{2}, v_{2}}\right)\right)^{\left(1+\gamma_{2}\right)}} d \mu_{2}(t) \\
& \leq C \mu_{1}\left(Q_{\tau_{1}}^{\mu_{1}, v_{1}}\right)^{-1 / 2} \mu_{2}\left(Q_{\tau_{2}}^{\mu_{2}, v_{2}}\right)^{-1 / 2} \chi_{W_{1}} \chi_{W_{2}} \\
& \times 2^{-\left(\mu_{1}-k_{1}\right)\left(1+\gamma_{1}\right)} 2^{-\left(\mu_{2}-k_{2}\right)\left(1+\gamma_{2}\right)} \\
& \leq C \mu_{1}\left(Q_{\tau_{1}}^{\mu_{1}, v_{1}}\right)^{-1 / 2} \mu_{2}\left(Q_{\tau_{2}}^{\mu_{2}, v_{2}}\right)^{-1 / 2} \\
& \times \frac{2^{-\left(\mu_{1}-k_{1}\right)\left(1+\gamma_{1}\right)}}{\left(1+2^{k_{1}} \rho_{1}\left(x, z_{\tau_{1}}^{\mu_{1}, v_{1}}\right)\right)^{1+\gamma_{1}}} \\
& \times \frac{2^{-\left(\mu_{2}-k_{2}\right)\left(1+\gamma_{2}\right)}}{\left(1+2^{k_{2}} \rho_{2}\left(y, z_{\tau_{2}}^{\mu_{2}, v_{2}}\right)\right)^{1+\gamma_{2}}},
\end{aligned}
$$

which is also a desired estimate.

By an analogou argument to $(48)_{4}$, we can verify $(48)_{5}$. We now prove $(49)_{1}$

$$
\begin{aligned}
& \left|D_{k_{1}} D_{k_{2}}\left(m_{R}\right)(x, y)\right| \\
& \quad=\left|\int_{X_{1} \times X_{2}} D_{k_{1}}(x, s) D_{k_{2}}(y, t) m_{R}(s, t) d \mu_{1}(s) d \mu_{2}(t)\right|
\end{aligned}
$$

$$
\begin{aligned}
& \leq \int_{X_{1} \times X_{2}}\left|D_{k_{1}}(x, s)\right|\left|D_{k_{2}}(y, t)-D_{k_{2}}\left(y, z_{\tau_{2}}^{\left(\mu_{2}, v_{2}\right)}\right)\right| \\
& \times\left|m_{R}(s, t)-m_{R}(x, t)\right| d \mu_{1}(s) d \mu_{2}(t) \\
&=\left(\chi_{w_{2}} \int_{X_{3} \times W_{4}}+\chi_{w_{2}} \int_{X_{3} \times W_{4}^{C}}+\chi_{W_{2}}^{C} \int_{X_{3} \times W_{4}}\right) \\
& \times\left|D_{k_{1}}(x, s)\right|\left|D_{k_{2}}(y, t)-D_{k_{2}}\left(y, z_{\tau_{2}}^{\left(\mu_{2}, v_{2}\right)}\right)\right| \\
&\left|m_{R}(s, t)-m_{R}(x, t)\right| d \mu_{1}(s) d \mu_{2}(t) \\
&:=(49)_{1}+(49)_{2}+(49)_{3} .
\end{aligned}
$$

For $(49)_{1}$, we have

$\left|(49)_{1}\right|$

$$
\begin{aligned}
& \leq \chi_{W_{2}} 2^{k_{1}} 2^{k_{2}\left(1+\epsilon_{2}\right)} \mu_{1}\left(Q_{\tau_{1}}^{\mu_{1}, v_{1}}\right)^{-1 / 2-\beta_{1}} \mu_{2}\left(Q_{\tau_{2}}^{\mu_{2}, v_{2}}\right)^{-1 / 2} \\
& \times \int_{W_{3} \times W_{4}} \rho_{1}(x, s)^{\beta_{1}}\left(\frac{1}{\left(1+2^{\mu_{1}} \rho_{1}\left(x, z_{\tau_{1}}^{\mu_{1}, v_{1}}\right)\right)^{1+\gamma_{1}}}\right. \\
&\left.+\frac{1}{\left(1+2^{\mu_{1}} \rho_{1}\left(s, z_{\tau_{1}}^{\mu_{1}, v_{1}}\right)\right)^{1+\gamma_{1}}}\right)
\end{aligned}
$$

$$
\times \frac{\rho_{2}\left(t, z_{\tau_{2}}^{\mu_{2}, v_{2}}\right)^{\epsilon_{2}}}{\left(1+2^{\mu_{2}} \rho_{2}\left(t, z_{\tau_{2}}^{\mu_{2}, v_{2}}\right)\right)^{1+\gamma_{2}}} d \mu_{1}(s) d \mu_{2}(t)
$$

$$
\begin{aligned}
& \leq \chi_{W_{2}} 2^{k_{1}} 2^{k_{2}\left(1+\epsilon_{2}\right)} \\
& \times \mu_{1}\left(Q_{\tau_{1}}^{\mu_{1}, v_{1}}\right)^{-1 / 2-\beta_{1}} \mu_{2}\left(Q_{\tau_{2}}^{\mu_{2}, v_{2}}\right)^{-1 / 2} \\
& \times \int_{W_{3}}\left(\frac{\rho_{1}(x, s)^{\beta_{1}}}{\left(1+2^{\mu_{1}} \rho_{1}\left(x, z_{\tau_{1}}^{\mu_{1}, v_{1}}\right)\right)^{1+\gamma_{1}}}\right. \\
& \left.+\frac{\rho_{1}(x, s)^{\beta_{1}}}{\left(1+2^{\mu_{1}} \rho_{1}\left(s, z_{\tau_{1}}^{\mu_{1}, v_{1}}\right)\right)^{1+\gamma_{1}}}\right) d \mu_{1}(s) \\
& \times \int_{\rho_{2}\left(t, z_{\tau_{2}}^{\mu_{2}, v_{2}}\right) \leq 8 C_{2} 2^{-k_{2}}} 2^{-\mu_{2}\left(1+\gamma_{2}\right)} \\
& \times \rho_{2}\left(t, z_{\tau_{2}}^{\mu_{2}, v_{2}}\right)^{\epsilon_{2}-1-\gamma_{2}} d \mu_{2}(t) \\
& \leq C \mu_{1}\left(Q_{\tau_{1}}^{\mu_{1}, v_{1}}\right)^{-1 / 2} \mu_{2}\left(Q_{\tau_{2}}^{\mu_{2}, v_{2}}\right)^{-1 / 2} \\
& \times \frac{2^{-\left(\mu_{2}-k_{2}\right)\left(1+\gamma_{2}\right)}}{\left(1+2^{k_{2}} \rho_{2}\left(y, z_{\tau_{2}}^{\mu_{2}, v_{2}}\right)\right)^{1+\gamma_{2}}} \\
& \times\left(2^{\mu_{1} \beta_{1}+k_{1}}\right. \\
& \times \int_{\rho_{1}(x, s) \leq 4 C_{1} 2^{-k_{1}}} \frac{\rho_{1}(x, s)^{\beta_{1}}}{\left(1+2^{\mu_{1}} \rho_{1}\left(s, z_{\tau_{1}}^{\mu_{1}, v_{1}}\right)\right)^{1+\gamma_{1}}} d \mu_{1}(s)
\end{aligned}
$$




$$
\begin{gathered}
\left.+\frac{2^{-\left(k_{1}-\mu_{1}\right) \beta_{1}}}{\left(1+2^{\mu_{1}} \rho_{1}\left(x, z_{\tau_{1}}^{\mu_{1}, \nu_{1}}\right)\right)^{1+\gamma_{1}}}\right) \\
\leq C \mu_{1}\left(Q_{\tau_{1}}^{\mu_{1}, v_{1}}\right)^{-1 / 2} \mu_{2}\left(Q_{\tau_{2}}^{\mu_{2}, v_{2}}\right)^{-1 / 2} \\
\times \frac{2^{-\left(k_{1}-\mu_{1}\right) \beta_{1}}}{\left(1+2^{\mu_{1}} \rho_{1}\left(x, z_{\tau_{1}}^{\mu_{1}, v_{1}}\right)\right)^{1+\gamma_{1}}} \\
\times \frac{2^{-\left(\mu_{2}-k_{2}\right)\left(1+\gamma_{2}\right)}}{\left(1+2^{k_{2}} \rho_{2}\left(y, z_{\tau_{2}}^{\mu_{2}, v_{2}}\right)\right)^{1+\gamma_{2}}},
\end{gathered}
$$

since $1+2^{\mu_{1}} \rho_{1}\left(x, z_{\tau_{1}}^{\mu_{1}, v_{1}}\right) \leq 2\left(2 C_{1}+1\right)\left(1+2^{\mu_{1}} \rho_{1}\left(s, z_{\tau_{1}}^{\mu_{1}, v_{1}}\right)\right)$ by $k_{1}>\mu_{1}$, which is the proof of $(48)_{1}$.

The verification of terms $(49)_{2}$ and $(49)_{3}$ is similar to that of (49) ${ }_{1}$ and proof of (50) is similar to that of (49) by symmetry.

We now prove (51). In this case, we have

$$
\begin{aligned}
& \left|D_{k_{1}} D_{k_{2}}\left(m_{R}\right)(x, y)\right| \\
& \leq \int_{X_{1} \times X_{2}}\left|D_{k_{1}}(x, s)\right|\left|D_{k_{2}}(y, t)\right| \\
& \times \mid m_{R}(s, t)-m_{R}(x, t) \\
& -m_{R}(s, y)-m_{R}(x, y) \mid d \mu_{1}(s) d \mu_{2}(t) \\
& \leq 2^{k_{1}} 2^{k_{2}} \mu_{1}\left(Q_{\tau_{1}}^{\mu_{1}, v_{1}}\right)^{-1 / 2-\beta_{1}} \mu_{2}\left(Q_{\tau_{2}}^{\mu_{2}, v_{2}}\right)^{-1 / 2-\beta_{2}} \\
& \times \int_{W_{3}} \rho_{1}(x, s)^{\beta_{1}}\left(\frac{1}{\left(1+2^{\mu_{1}} \rho_{1}\left(x, z_{\tau_{1}}^{\mu_{1}, v_{1}}\right)\right)^{1+\gamma_{1}}}\right. \\
& \left.+\frac{1}{\left(1+2^{\mu_{1}} \rho_{1}\left(s, z_{\tau_{1}}^{\mu_{1}, v_{1}}\right)\right)^{1+\gamma_{1}}}\right) d \mu_{1}(s) \\
& \times \int_{W_{4}} \rho_{2}(y, t)^{\beta_{2}}\left(\frac{1}{\left(1+2^{\mu_{2}} \rho_{2}\left(y, z_{\tau_{2}}^{\mu_{2}, v_{2}}\right)\right)^{1+\gamma_{2}}}\right. \\
& \left.+\frac{1}{\left(1+2^{\mu_{2}} \rho_{2}\left(t, z_{\tau_{2}}^{\mu_{1}, v_{2}}\right)\right)^{1+\gamma_{2}}}\right) d \mu_{2}(t) \\
& \leq C \mu_{1}\left(Q_{\tau_{1}}^{\mu_{1}, v_{1}}\right)^{-1 / 2} \mu_{2}\left(Q_{\tau_{2}}^{\mu_{2}, v_{2}}\right)^{-1 / 2} \\
& \times\left(\frac{2^{-\left(k_{1}-\mu_{1}\right) \beta_{1}}}{\left(1+2^{\mu_{1}} \rho_{1}\left(x, z_{\tau_{1}}^{\mu_{1}, \nu_{1}}\right)\right)^{1+\gamma_{1}}}\right. \\
& \left.+2^{\mu_{1} \beta_{1}+k_{1}} \int_{W_{3}} \frac{\rho_{1}(x, s)^{\beta_{1}}}{\left(1+2^{\mu_{1}} \rho_{1}\left(s, z_{\tau_{1}}^{\mu_{1}, v_{1}}\right)\right)^{1+\gamma_{1}}} d \mu_{1}(s)\right)
\end{aligned}
$$

$$
\begin{aligned}
& \times\left(\frac{2^{-\left(k_{2}-\mu_{2}\right) \beta_{2}}}{\left(1+2^{\mu_{2}} \rho_{2}\left(y, z_{\tau_{2}}^{\mu_{2}, v_{2}}\right)\right)^{1+\gamma_{2}}}\right. \\
& \left.\quad+2^{\mu_{2} \beta_{2}+k_{2}} \int_{W_{4}} \frac{\rho_{2}(y, t)^{\beta_{2}}}{\left(1+2^{\mu_{2}} \rho_{2}\left(t, z_{\tau_{2}}^{\mu_{2}, v_{2}}\right)\right)^{1+\gamma_{2}}} d \mu_{2}(t)\right) \\
& \leq C \mu_{1}\left(Q_{\tau_{1}}^{\mu_{1}, v_{1}}\right)^{-1 / 2} \mu_{2}\left(Q_{\tau_{2}}^{\mu_{2}, v_{2}}\right)^{-1 / 2} \\
& \times \frac{2^{-\left(k_{1}-\mu_{1}\right) \beta_{1}}}{\left(1+2^{\mu_{1}} \rho_{1}\left(x, z_{\tau_{1}}^{\mu_{1}, v_{1}}\right)\right)^{1+\gamma_{1}}} \\
& \times \frac{2^{-\left(k_{2}-\mu_{2}\right) \beta_{2}}}{\left(1+2^{\mu_{2}} \rho_{2}\left(y, z_{\tau_{2}}^{\mu_{2}, v_{2}}\right)\right)^{1+\gamma_{2}}} .
\end{aligned}
$$

\section{Conflict of Interests}

The authors declare that there is no conflict of interests regarding the publication of this paper.

\section{Acknowledgments}

The authors would like to thank the referees for carefully reading the paper and for making valuable comments. The first and second authors are supported by NNSF, China (Grant no. 11171345) and the Doctoral Fund of Ministry of Education of China (Grant no. 20120023110003). The second author is also supported by the NNSF, China (Grant no. 51234005). The third author is supported by the Fundamental Research Funds for the Central Universities (Grant no. JCB2013B06).

\section{References}

[1] R. R. Coifman, "A real variable characterization of $H^{p}$," Studia Mathematica, vol. 51, pp. 269-274, 1974.

[2] R. H. Latter, "A characterization of $H^{p}\left(\mathbb{R}^{n}\right)$ in terms of atoms," Studia Mathematica, vol. 62, no. 1, pp. 93-101, 1978.

[3] R. R. Coifman, "Characterization of Fourier transforms of Hardy spaces," Proceedings of the National Academy of Sciences of the United States of America, vol. 71, pp. 4133-4134, 1974.

[4] R. R. Coifman and G. Weiss, "Extensions of Hardy spaces and their use in analysis," Bulletin of the American Mathematical Society, vol. 83, no. 4, pp. 569-645, 1977.

[5] S.-Y. A. Chang and R. Fefferman, "A continuous version of the duality of $H^{1}$ and BMO on the bidisk," Annals of Mathematics, vol. 112, no. 1, pp. 179-201, 1980.

[6] S. A. Chang and R. Fefferman, "The Calderón-Zygmund decomposition on product domains," The American Journal of Mathematics, vol. 104, no. 3, pp. 455-468, 1982.

[7] Y. Han, G. Lu, and D. Yang, Product theory on spaces of homogeneous type, unpublished manuscript.

[8] Y. Han, G. Lu, and K. Zhao, “Discrete Calderón’s identity, atomic decomposition and boundedness criterion of operators on 
multiparameter Hardy spaces," Journal of Geometric Analysis, vol. 20, no. 3, pp. 670-689, 2010.

[9] M. Frazier and B. Jawerth, "A discrete transform and decompositions of distribution spaces," Journal of Functional Analysis, vol. 93, no. 1, pp. 34-170, 1990.

[10] Y. S. Han and E. T. Sawyer, "Littlewood-Paley theory on spaces of homogeneous type and the classical function spaces," Memoirs of the American Mathematical Society, vol. 110, no. 530, pp. 1-126, 1994.

[11] Y. Han, S. Lu, and D. Yang, "Inhomogeneous Besov and TriebelLizorkin spaces on spaces of homogeneous type," Approximation Theory and its Applications, vol. 15, no. 3, pp. 37-65, 1999.

[12] W. Wang, "A discrete transform and Triebel-Lizorkin spaces on the bidisc," Transactions of the American Mathematical Society, vol. 347, no. 4, pp. 1351-1364, 1995.

[13] Y. Han, C. Lin, G. Lu, Z. Ruan, and E. T. Sawyer, "Hardy spaces associated with different homogeneities and boundedness of composition operators," Revista Matemática Iberoamericana, vol. 29, no. 4, pp. 1127-1157, 2013.

[14] X. Wu, "Boundedness of composition of operators associated with different homogeneities on weighted Besov and TriebelLizorkin spaces," Proceedings of the Indian Academy of Sciences, Mathematical Sciences, vol. 124, no. 1, pp. 81-92, 2014.

[15] Y. Han, J. Li, and G. Lu, "Multiparameter Hardy space theory on CARnot-Carathéodory spaces and product spaces of homogeneous type," Transactions of the American Mathematical Society, vol. 365, no. 1, pp. 319-360, 2013. 


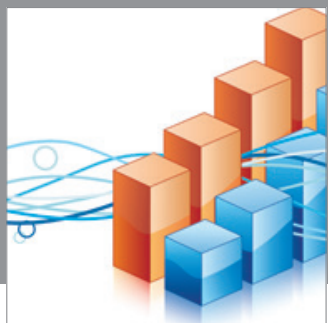

Advances in

Operations Research

mansans

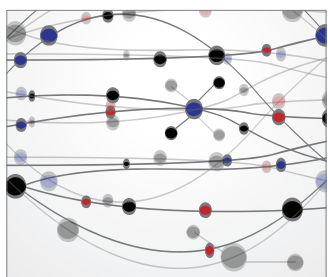

The Scientific World Journal
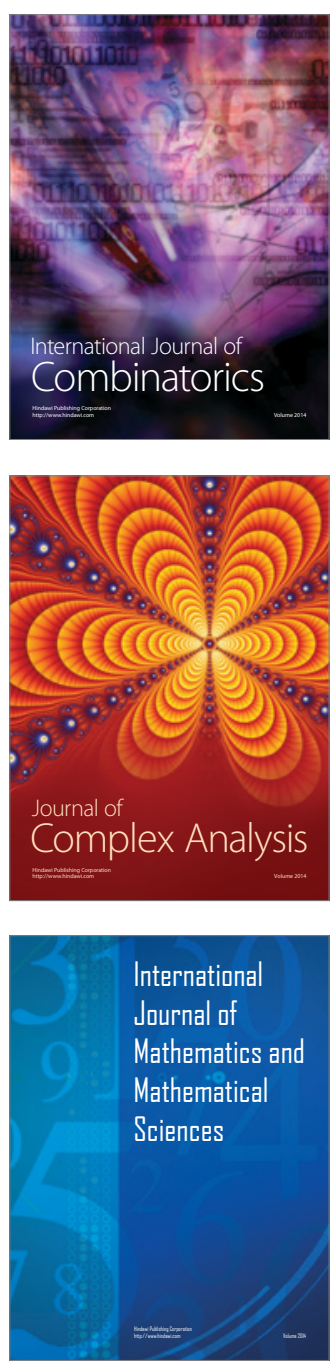
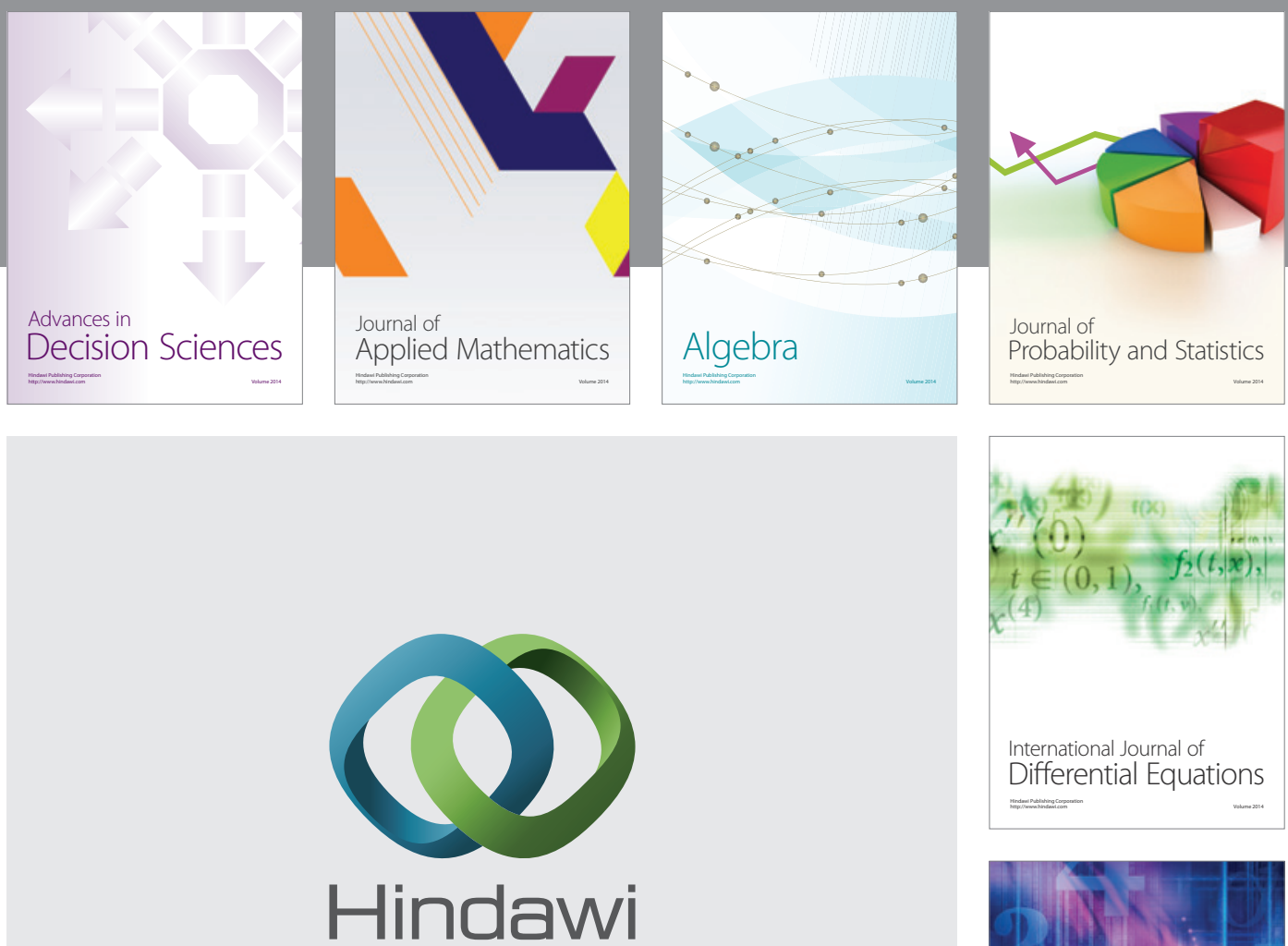

Submit your manuscripts at http://www.hindawi.com
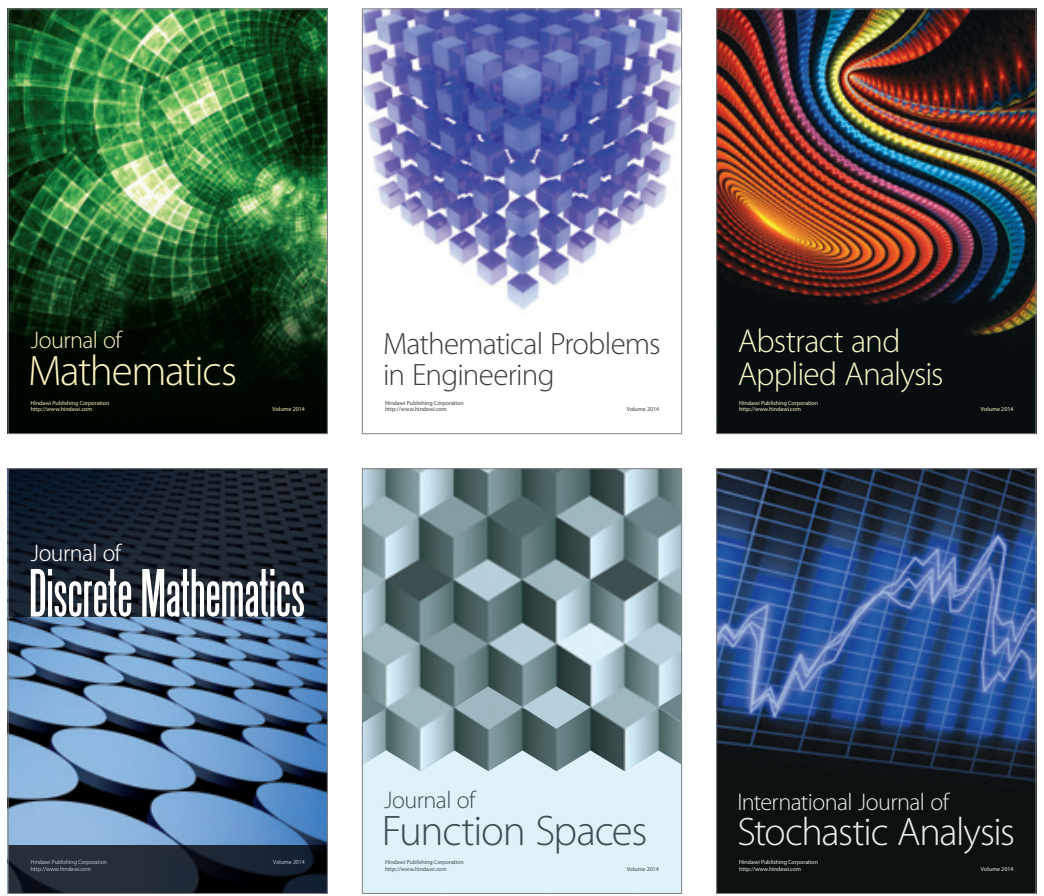

Journal of

Function Spaces

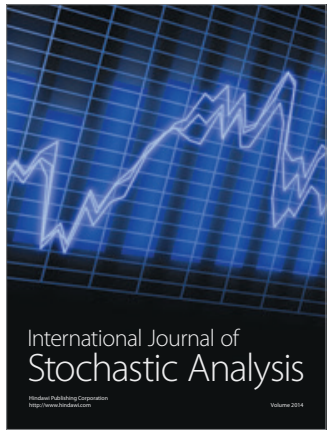

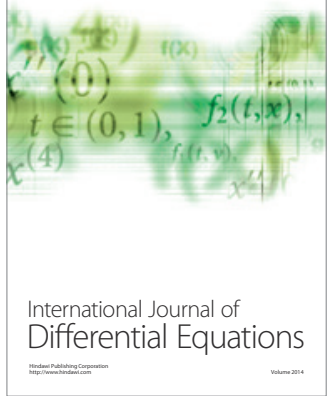
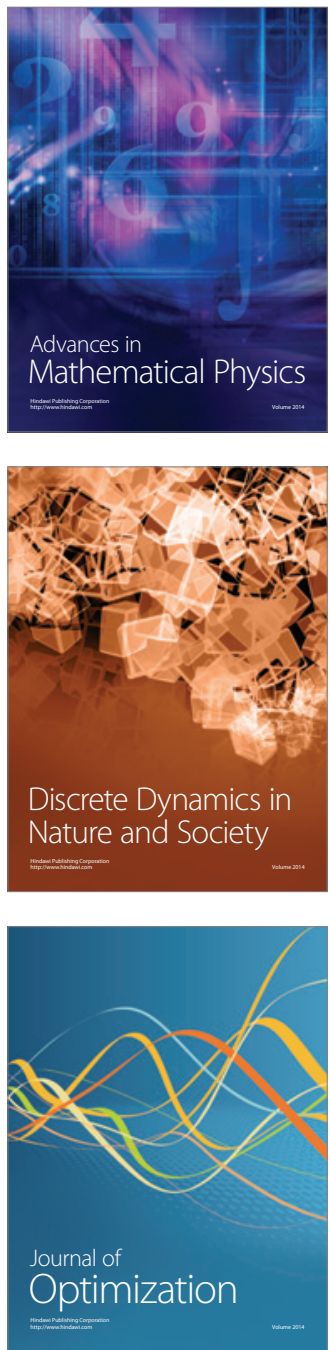